\title{
Dithen: A Computation-as-a-Service Cloud Platform For Large-Scale Multimedia Processing
}

\author{
Joseph Doyle, Vasileios Giotsas, Mohammad Ashraful Anam and Yiannis Andreopoulos, Senior Member, IEEE
}

\begin{abstract}
We present Dithen, a novel computation-as-a-service (CaaS) cloud platform specifically tailored to the parallel execution of large-scale multimedia tasks. Dithen handles the upload/download of both multimedia data and executable items, the assignment of compute units to multimedia workloads, and the reactive control of the available compute units to minimize the cloud infrastructure cost under deadline-abiding execution. Dithen combines three key properties: (i) the reactive assignment of individual multimedia tasks to available computing units according to availability and predetermined time-to-completion constraints; (ii) optimal resource estimation based on Kalmanfilter estimates; (iii) the use of additive increase multiplicative decrease (AIMD) algorithms (famous for being the resource management in the transport control protocol) for the control of the number of units servicing workloads. The deployment of Dithen over Amazon EC2 spot instances is shown to be capable of processing more than 80,000 video transcoding, face detection and image processing tasks (equivalent to the processing of more than 116 GB of compressed data) for less than $\$ 1$ in billing cost from EC2. Moreover, the proposed AIMD-based control mechanism, in conjunction with the Kalman estimates, is shown to provide for more than $27 \%$ reduction in EC2 spot instance cost against methods based on reactive resource estimation. Finally, Dithen is shown to offer a $38 \%$ to $500 \%$ reduction of the billing cost against the current state-of-the-art in CaaS platforms on Amazon EC2 (Amazon Lambda and Amazon Autoscale). A baseline version of Dithen is currently available at dithen.com.
\end{abstract}

Index Terms-computation-as-a-service, big data, multimedia computing, cloud computing, Amazon EC2, spot instances.

\section{INTRODUCTION}

I NFRASTRUCTURE-AS-A-SERVICE (IaaS) providers, such as Amazon Elastic Compute Cloud (EC2), Google Compute Engine (GCE), IBM Bluemix and Rackspace, now allow for the flexible reservation of compute units (CUs) in the cloud (i.e., pre-established sets of processor cores, memory, storage and operating systems), with yearly, daily, hourly or even minute-by-minute billing [1], [2]. This has led to the explosion of Platform-as-a-Service (PaaS) and Software-as-aService (SaaS) offerings [3], [4]. Within PaaS systems, the user is able to develop and execute multimedia processing tasks on distributed computing environments (e.g., Apache Hadoop

The authors are with Dithen, 843 Finchley Road, London, NW11 8NA, U.K., dithen.com; email: \{j.doyle, v.giotsas, russell.anam, i.andreopoulos $\} @$ dithen.com. JD is also with the University of East London, University Way, London, E16 2RD, U.K. MAA and YA are also with the Electronic and Electrical Engineering Department, University College London, Roberts Building, Torrington Place, London, WC1E 7JE, UK. VG is also with the Center for Applied Internet Data Analysis, University of California at San Diego, CAIDA UCSD/SDSC, 9500 Gilman Dr., La Jolla, CA, 92093-0505. This work was supported in part by Innovate UK (feasibility project ACAME, 131983). and mesos, Google App Engine, Microsoft Azure, etc.) on IaaS providers, albeit at the cost of converting the multimedia processing software to code that can be scaled-up by the PaaS infrastructure (for example converting the operations to Map and Reduce steps in Hadoop). In SaaS, a provider licenses a specific set of applications to customers (e.g., pre-established word processing software, a fixed set of video transcoding or video streaming toolboxes, etc.) either as a service on demand, through a subscription, or in a pay-as-you-go model [5]. In the multimedia systems domain, this provides the opportunity to use transcoding or signal processing algorithms and toolboxes directly [4], [6]-[10], and has led to the development of related commercial services.

\section{A. From Platform and Software-as-a-Service to Computation- as-a-Service}

This evolution of IaaS, PaaS and SaaS is now beginning to lead to Computation-as-a-Service (CaaS) [11], where users can upload multimedia (e.g., image, audio or video) files and scripts or binary files prepared in their local environment [4], [6], [10], [12], [13] in order to be executed in CUs in the cloud directly, i.e., without having to develop and manage any infrastructure or convert their software to a format amenable to distributed computing environments. CaaS provides a useful compromise between the generality of IaaS and PaaS offerings and the ease-of-use of SaaS: the end user can deploy and scale any desktop multimedia application of their choosing without needing to adapt its codebase. This differs from the case of $\mathrm{SaaS}$, in that the user can simply execute any Matlab, $\mathrm{C} / \mathrm{C}++$, Java, OpenCV, Javascript/Python based code and scripts of their local platform on the CaaS platform without any modification by following a simple set of rules. The CaaS platform can then handle the scheduling and parallelization of multiple multimedia workloads without any user intervention via the appropriate reservation (or bidding) of resources from IaaS providers, e.g., Amazon EC2 spot instance bidding or GCE CU reservation.

\section{B. Related Work}

Workload scheduling on CaaS systems has some resemblance to well-studied scheduling problems for large computing clusters [14]-[16]. However, two major differences between the two domains are that resources of cluster computing systems are persistent and "prepaid", i.e., the number of CUs does not fluctuate during the execution of a workload and there is no penalty for unused CUs. On the other hand, because CaaS resources are billed according to compute instance reservation, 
a CaaS provider often initiates or terminates CUs during the execution of a workload in order to minimize the monetary cost incurred, while abiding to the agreed workload completion time. To this end, there have been numerous recent proposals for cloud resource management. Gandhi et. al. propose their own version of Autoscale, which stops servers that have been idle for more than a specified time, while concentrating jobs on less CUs to reduce cost [17]. Paya et. al. expand on this by proposing a system that uses multiple sleep states to improve performance [18]. Song et. al. propose optimal allocation of CUs according to pricing and demand distributions [1]. Ranjan et. al. investigate architectural elements of content-delivery networks with cloud-computing support [19]. Jung et. al. propose multi-user workload scheduling on various CUs based on genetic algorithms [20]. Beyond resource allocation and scheduling, a major challenge in CaaS frameworks is the varying delay in the completion of various multimedia processing workloads [4], [21]. The processing delay primarily depends on: the workload specifics, the CU reservation mechanism employed, and the transport-layer jitter (if data is continuously transported to/from users and cloud providers) [5]. This is the primary reason why all real-world CaaS platforms only provide "best effort" service level agreements (SLAs) for large workload execution without considering a predetermined timeto-completion (TTC) estimate. Recent research work on this front proposes the use of particle swarm optimisation to derive viable schedules [22] and the use of the earliest-deadline first algorithm [23]. While all such proposals are effective in their resource provisioning for TTC-abiding execution, they assume that the system has accurate estimates of the computation required to complete each workload. However, this is unlikely to be the case in practice, particularly at the start of a workload's execution. Therefore, our proposal considers the realistic scenario where no estimates for the computational requirements are available at the start of each workload's execution; i.e., in conjunction with resource provisioning, our framework performs an adaptive resource estimation during the execution of each workload.

Finally, the first commercial CaaS offerings are now beginning to emerge. The key representatives are: (i) the recentlyannounced AWS Lambda service, where users can submit individual Javascript items and be billed at a fixed rate per $100 \mathrm{~ms}$ of Lambda service usage under a best-effort SLA; (ii) PiCloud, a service for flexible scheduling of batch processing tasks via a terminal command line interface; (iii) Parse, a software development environment for Javascript execution on cloudcomputing infrastructures; and (iv) Amazon EC2 Autoscale, a service that automatically scales application deployment over Amazon EC2 according to processor and network utilization constraints. In all these deployments, the comparative metric for workload analysis is the required processing time in terms of the number of seconds a single core was occupied until the workload is successfully completed. We therefore quantify the resource reservation in the IaaS provider via compute-unit seconds (CUSs), i.e., the product of the total cores used with the time they were reserved for, since charges will be applied for them from the IaaS provider regardless of whether the CaaS system actually used them to their full capacity or not.

\section{Contribution}

While the current research and commercial efforts in CaaS frameworks are a promising start, they do not consider the reactive estimation of the required CUSs to process submitted workloads, or assume that the CUS metric per workload is known [4], [22], [23]. In addition, current CaaS frameworks do not consider on-demand CU provisioning (e.g., EC2 spot instances or GCE CUs with minute-level increments) under TTC constraints, where it is imperative to control both the allocation and termination of new instances in order to reduce the infrastructure cost while providing for TTC-abiding execution. Finally, at the moment there are very limited options for CaaS frameworks to develop and benchmark multimedia cloudcomputing services and the multimedia systems community would benefit from new efforts on this front.

In this light, we present Dithen, a new cloud computing service that scales small and medium-level execution of data processing workloads to big data under TTC constraints. For example, algorithms for video transcoding, image classification, object recognition, etc., that run on small volumes of input images/videos on a desktop computing system can be directly scaled-up via Dithen (i.e., without any code modifications) to operate on big datasets comprising millions of input images and videos, with a-priori established completion times. Dithen meets the requirements for such large-scale dataintensive processing by combining the following novel aspects:

1) It supports the direct upload and execution of bash, Python, Java, Javascript and Matlab scripts, as well as the execution of statically-built binaries for 32-bit or 64bit Ubuntu Linux or Microsoft Windows on any number of EC2 spot instances.

2) Each submitted workload is separated into individuallyexecutable tasks, which are then allocated to available CUs with proportionally-fair scheduling in order to: (i) maximize the available $\mathrm{CU}$ utilization and (ii) abide by the confirmed TTC value for the workload. The finegrain partitioning of each workload into tasks allows for each user to check that the output results are being produced correctly by Dithen during execution and cancel the workload execution if otherwise.

3) Estimates of the required CUSs until the completion of each task type in each workload are derived based on Kalman-filter estimators, which are shown to significantly outperform other ad-hoc estimators.

4) Based on the estimation of the required CUSs, Dithen uses the Additive Increase Multiplicative Decrease (AIMD) algorithm [24] for the allocation or termination of CUs according to the expected workload. While AIMD is a well-known control mechanism for network resource utilization, e.g., within the transport control protocol (TCP), to the best of our knowledge, this is the first time it is proposed for CaaS provisioning.

Finally, beyond describing Dithen, we also provide free access to it $^{1}$ at dithen.com.

${ }^{1}$ each new user account gets an amount of free credit to spend on the service 
TABLE I

Nomenclature And Notational Conventions.

\begin{tabular}{|c|c|}
\hline Key Concept & Definition \\
\hline$t$ & monitoring time instant \\
\hline$W[t]$ & total workloads in Dithen at time instant $t$ \\
\hline$M[t]$ & total media types at time $t$ \\
\hline$m_{w, k}[t]$ & $\begin{array}{l}\text { remaining media items of type } k \text { to be processed } \\
\text { within workload } w \text { at time } t(1 \leq k \leq M[t] \\
1 \leq w \leq W[t]\end{array}$ \\
\hline$I$ & total types of instances in the cloud infrastructure \\
\hline$p_{i}$ & $\begin{array}{l}\text { compute units (CUs), i.e., processor cores, available } \\
\text { within instance type } i, 1 \leq i \leq I\end{array}$ \\
\hline$n_{i}[t], N_{\mathrm{tot}}$ & $\begin{array}{l}\text { number of instances of type } i(1 \leq i \leq I) \text { reserved } \\
\text { at time } t \text {, total number of CUs in Dithen }\end{array}$ \\
\hline$a_{i, j}[t]$ & $\begin{array}{l}\text { remaining time for the } j \text { th instance of type } i \text { before } \\
\text { additional billing is incurred by the cloud provider }\end{array}$ \\
\hline$c_{\mathrm{tot}}[t], c_{\min }, c_{\max }$ & $\begin{array}{l}\text { total compute-unit-seconds (CUSs) available in } \\
\text { Dithen, and lower/upper limits for CUSs in Dithen }\end{array}$ \\
\hline$d_{w}[t]$ & time-to-completion (TTC) for workload $w$ at time $t$ \\
\hline$\hat{b}_{w, k}[t]$ & $\begin{array}{l}\text { CUS estimate to process a media item of type } k \text { of } \\
\text { workload } w\end{array}$ \\
\hline$r_{w}[t]$ & required CUSs for the completion of workload $w$ \\
\hline$s_{w}[t]$ & service rate, i.e., CUs allocated for workload $w$ \\
\hline$z_{w, k}[t], v_{w, k}[t]$ & $\begin{array}{l}\text { CUS process and measurement noise instantiations of } \\
\text { media type } k \text { of workload } w\end{array}$ \\
\hline$\alpha, \beta$ & $\begin{array}{l}\text { additive increase and multiplicative decrease } \\
\text { parameters of AIMD }\end{array}$ \\
\hline Notation & Explanation \\
\hline $\begin{array}{c}\text { uppercase } \\
\text { Roman letters }\end{array}$ & random variables \\
\hline $\begin{array}{l}\text { lowercase } \\
\text { Greek letters }\end{array}$ & $\begin{array}{l}\text { moments of probability distributions, stochastic } \\
\text { parameters of Kalman filters, or AIMD and ARMA } \\
\text { parameters }\end{array}$ \\
\hline$\tilde{b}$ & measurement of quantity $b$ \\
\hline$\hat{b}$ & estimation of quantity $b$ \\
\hline
\end{tabular}

The remainder of this paper is organized as follows. Section II presents the architecture of Dithen. Sections III and IV present the key elements of the proposed CUS estimation and AIMD framework, while Section V presents experimental results and comparisons of different $\mathrm{CU}$ allocation strategies for Amazon EC2 spot instances. Finally, Section VI presents some concluding remarks.

\section{Anatomy of the Dithen Architecture}

The architecture of Dithen is pictorially illustrated in Fig. 1. It comprises five elements: the Front End (FE), the Cloud Storage and Instance Types (CS-IT), the Monitoring Element (ME), and the Local and Global Controller Instances (LCI and GCI). Their functionality is detailed in the following subsections. To aid the exposition, Table I summarizes the nomenclature and notational conventions used.

\section{A. Overview of the Operation of Dithen}

In order to illustrate the roles of the different components of our system, we first present an overview of how Dithen

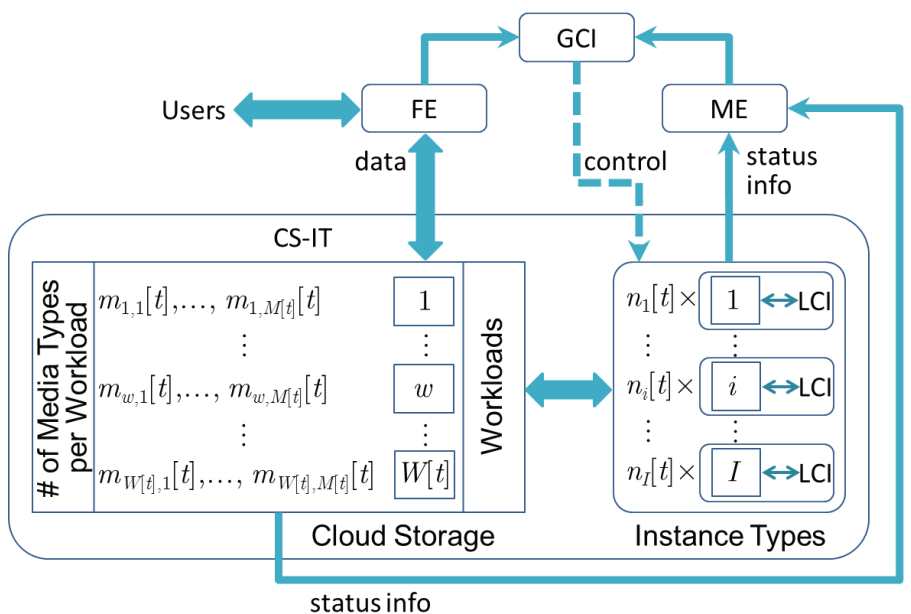

Fig. 1. Dithen architecture. At each monitoring instant $t$, each workload $w(1 \leq w \leq W[t])$ contains several media types. In addition, $n_{i}[t]$ spot instances of type $i(1 \leq i \leq I)$ are reserved and can be used to process workloads.

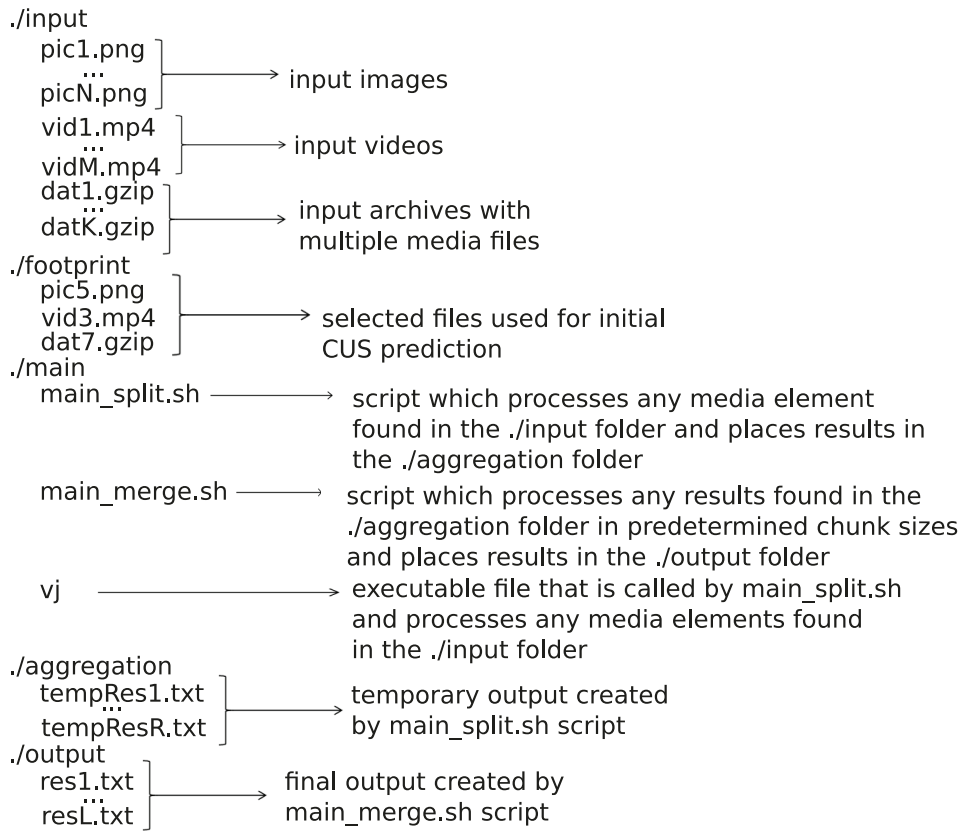

Fig. 2. Example workload structure.

processes a workload. A user submits a workload via the FE (Section II-B) and can request a particular TTC value for this workload, or a TTC is allocated by Dithen. As shown in Fig. 2, the workload may comprise multiple input media files (e.g., JPEG images, MP4 video files, etc.), as well as scripts and executable files to process the inputs. An executable file can be a Linux or Windows binary and the script can be a Linux shell script, Windows batch file or Matlab/Octave script file. Dithen typically handles workloads where each input is processed independently from the other inputs. This is the norm for large-scale multimedia cloud computing where a user typically wants to carry out a certain task (e.g., face recognition, transcoding, etc.) on a large cache of input images or videos. Nevertheless, as explained in later sections (and as 
shown in Fig. 2) it is also possible to: (i) package multiple inputs together in a single input archive (e.g., gzip file, the contents of which are automatically extracted by Dithen in the utilized CU prior to processing) for concurrent processing of batches of input media, (ii) execute "Split-Merge" tasks akin to MapReduce code, i.e., code that performs parallel processing of inputs (Split step) followed by aggregation of multiple outputs in order to produce the final results (Merge step).

Once the GCI detects that a new workload has been added, it assigns a small percentage of the inputs of the workload (e.g., $5 \%$ of the submitted inputs) to LCIs in a "footprinting" stage. The compute instances of the LCIs execute the submitted code on their assigned inputs and provide the corresponding execution times to the ME (Section II-D) and, via that, to the GCI. These measurements and the logs of the execution status (e.g., 0 for normal and -1 for abnormal termination) are used by GCI to: (i) confirm that the workload processing is carried out without errors or crashes in the submitted code; (ii) derive an initial Kalman-based estimate of the required CUSs to complete this workload (Section II-E-1). This estimate is used to confirm that the requested workload TTC is achievable by Dithen, or else adjust the confirmed TTC accordingly (Section II-E-2). The GCI continues to derive CUS estimates per workload in order to: (i) assign a service rate per workload, according to which all LCIs can process workload tasks via their corresponding instances (Section III); (ii) determine if the number of CUs should be scaled up or down (via the proposed AIMD algorithm of Section IV) so that all confirmed TTCs are met without excessive billing from the cloud provider. Finally, the results produced by all CUs are uploaded to Amazon Simple Storage Service (S3, see Section II-C), where they can be viewed by the user through the Dithen FE.

\section{B. Front End and Workload Processing Modes}

The FE of Dithen provides for workload uploading, launching, monitoring of execution, basic text file and image viewing and editing functionalities (e.g., for log file or launch script viewing and editing), and downloading of the results of individual tasks as they are being produced by CUs. Users can utilize the FE to cancel pending workloads if the results are deemed to be unsatisfactory or incorrectly executed (e.g., due to unsupported runtime components, or crashes/errors in the user's executed code). The simplicity of the FE of Dithen is evident from Fig. 3: the buttons allow for a point-and-click interface via a web browser. A basic mobile FE interface is also available.

1. Code \& data elements and baseline workload processing mode: In the basic mode of operation, Dithen assumes that the user provides a "main" bash/batch script (i.e., main.sh in Linux or main.bat in Microsoft Windows) for each application, which invokes all the required Matlab, Python, Java/Javascript code, or application binaries. All provided multimedia elements must be available in the folder . / input/ within each application. The results are produced in the locations created and specified within the user's own code (typically in application folders such as . / output/ or ./results/, etc.). The only constraint imposed by Dithen is that results must not be created within the input folder, which should be reserved solely for input files. An example of a typical input/output application structure is given in Fig. 3(a) and an example of the FE for the job monitoring is given in Fig. 3(b).

Each new user account created by the FE includes by default (at least) four example image processing applications that illustrate how to use the service: (i) Ex1_face_detection-automated detection of human faces within individual images using a stand-alone $\mathrm{C}++$ implementation of the Viola-Jones algorithm [25]; (ii) Ex2 template match-template matching between input images with an existing library of template images using the ImageMagick compare tool [26]; (iii) Ex3_image_merge-merging of groups of input JPEG files into a single animated GIF file using the ImageMagick convert tool [26]; (iv) Ex4_Matlab_SIFT-image salient point detection and description using the SIFT algorithm [27] via a Matlab implementation compiled to standalone binary with the Mathworks deploytool.

In all cases, the provided code utilizes each input media file independently and populates the output folder(s) with the results of the executed algorithm(s). Each such execution comprises a media processing task. The entire volume of independently-processed inputs, along with the user's application code, comprises a workload. If a number of media inputs (e.g., images, videos and/or audio files) must be processed together by the provided application code, they must be packaged into a single file container ${ }^{2}$ (e.g., TAR, ZIP or RAR). Dithen will automatically extract all such compressed format containers prior to calling the main script.

2. Advanced workload processing mode: To allow for more complex interactions between inputs and subsequent results, such as split and merge tasks that are similar to MapReduce code [28], Dithen provides an advanced workload processing mode, specifically designed to allow for the execution of Split-Merge tasks, e.g., the parallel execution of visual feature extraction from input JPEG images followed by aggregation of the produced feature points into a single feature matrix of reduced dimensions [29], [30]. This mode is triggered by the user uploading two "main" scripts, called main_split.sh and main_merge.sh (see also Fig. 2). When these scripts are found in the application folder, the first one is executed as in the basic mode of operation described previously but, instead of returning the results to the FE, it uploads them to a specially designated "aggregation" spot instance in Amazon EC2 that runs the second (i.e., "Merge") script. In this way, the latter script can invoke any aggregation code and the final results are provided to the FE. For example, within image retrieval or face recognition applications [29]-[31], the user can parallelize the computation of a very large number of image covariance matrices or vectors of local features via the "Split" script and then perform a large singular value decomposition (SVD) of the results (once they become available) by having the "Merge" script

\footnotetext{
${ }^{2}$ This is illustrated in example Ex3_image_merge.
} 


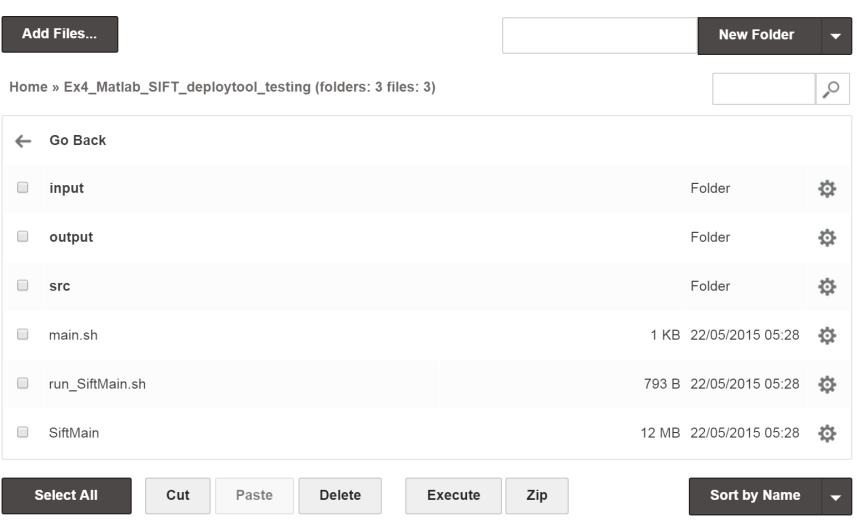

(a)

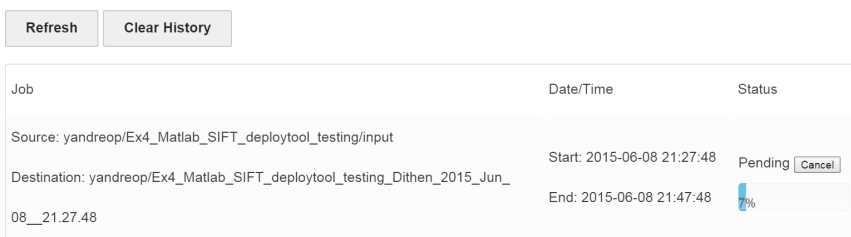

(b)

Fig. 3. (a) Example of front-end contents for a user workload executing the SIFT feature extraction on a large image dataset. The input image dataset is contained in the ./input folder and the output results are produced in the . / output folder. (b) Example of the workload execution indicating the source and destination folders and the confirmed TTC.

periodically poll for the full set of results of the Split step and invoke the SVD code on them. The data and executable items corresponding to such an example are illustrated in Fig. 2: the Split step is launched simultaneously by multiple instances running the main_split.sh script (each containing subsets of inputs) and each instance calculates one or more of the tempRes1.txt..tempResR.txt results and places them in the /aggregation folder of a specially-designated "Merge" instance. This instance is running the main_merge. sh script periodically in order to poll the /aggregation folder and, once sufficient outputs are detected, produces each of the res 1 . txt. . resL. txt files based on groups of such outputs. The rule of how many (and which) outputs to poll for, as well as the polling frequency (e.g., once per minute), is set by the user within the main_merge.sh script.

\section{Cloud Storage and Instance Types}

The CS-IT deployment depends on the possibilities available by the IaaS provider. In this paper, we evaluate Dithen with Amazon EC2 spot instances and Amazon S3 storage. We opt for EC2 spot instances as they provide for a wide variety of available configurations and for flexible billing based on hourly reservations. Future evaluations can incorporate other IaaS providers, like GCE, IBM Bluemix and Rackspace.

Dithen uses Ubuntu Linux and MS Windows spot instances that have been setup with bash shell support (or batch script file support for MS Windows), Python, Java, Javascript, Matlab (via the Mathworks Matlab Compiler Runtime), and OpenCV and ImageMagick library support. As shown in Fig. 1, de- pending on the type of scripts and executables submitted by the user, either of these instances can be spawned into any number of spot instances of type $i$, out of $I$ total instance types. This is performed by bidding for an appropriate spot instance type in Amazon's EC2 launch process (out of more than twenty types available) and specifying the reserved Amazon machine image id to be spawned. We denote the number of CUs per instance type by $p_{i}, 1 \leq i \leq I$. Moreover, at the $t$ th time instant, the Dithen architecture contains $n_{i}[t]$ total instances of type $i$. Finally, for the $j$ th instance out of the $n_{i}[t]$ ones, the remaining time until the next billing increment (e.g., until the time when the IaaS provider will bill for the next hour in the corresponding spot instance) is denoted by $a_{i, j}[t]$ (in seconds). Spot instances are requested using the requestSpotInstance () function which is part of the EC2 class of the AWS SDK. Instances are terminated using the terminateInstances () of the same class. Finally, the number of active spot instances is monitored using the describeInstances () function of the same class.

\section{Monitoring Element}

In order to observe the CU utilization, Dithen includes a monitoring element that measures processor utilization within each spot instance via the mpstat Linux command (or wmic cpu in MS Windows). Monitoring and reactive control of the execution of workloads take place at discrete "monitoring" time instants, typically every $1-5$ minutes. The ensemble of all the currently executing workloads within Dithen at the $t$ th monitoring instant includes $M[t]$ different media types (e.g., images, audio, video files, or container files comprising composite media and data types). The ME keeps track of a number of operational parameters described below (and summarized in Table I).

At every monitoring instant $t$, and within each workload $w$ $(1 \leq w \leq W[t])$, the ME keeps track of the number of remaining elements to be processed, $m_{w, k}[t]$, as well as the estimated CUSs required to complete the processing of each media type $k$ with the workload, $\hat{b}_{w, k}[t]$. The values of $m_{w, k}[t]$ are determined using an SQL database that records which tasks have been processed and the getIterator ( 'Listobjects') function of the S3 class of the AWS SDK. The estimates $\hat{b}_{w, k}[t]$ are derived based on the estimation process described in Subsection II-E-3. Typically, the SLA for each workload includes execution within a predetermined TTC value, $d_{w}[t]$, which is confirmed after an initial CUS estimate is available for the workload. To this end, the ME continuously keeps track of the required CUSs to complete each workload $w, r_{w}[t]$, which can be estimated by:

$$
r_{w}[t]=\sum_{k=1}^{M[t]} m_{w, k}[t] \hat{b}_{w, k}[t] .
$$

Finally, the ME keeps track of the total number of active CUs in Dithen by:

$$
N_{\text {tot }}[t]=\sum_{i=1}^{I} p_{i} n_{i}[t],
$$


as well as the total compute-unit seconds billed (i.e., already paid to the IaaS provider and available to use) within the Dithen architecture at any given instant $t$ :

$$
c_{\text {tot }}[t]=\sum_{i=1}^{I} \sum_{n=1}^{n_{i}[t]} p_{i} a_{i, n}[t] .
$$

Effectively, $c_{\text {tot }}[t]$ and $N_{\text {tot }}[t]$ represent a "snapshot" of the compute resources in Dithen at the $t$ th time instant, as they comprise the available CUSs and CUs under the already-billed EC2 instances.

\section{E. Local and Global Controller Instances}

The main tasks at every monitoring time instant $t$ are: (i) to ensure that each workload $w$ is executed within its confirmed TTC, $d_{w}[t]$, and (ii) to match $c_{\text {tot }}[t]$ to $\sum_{w=1}^{W[t]} r_{w}[t]$. Both must be met with the minimum billing from the IaaS. These two tasks are accomplished by the LCI and GCI components of Fig. 1, respectively. Towards this end, the most crucial aspects are: (i) defining reliable CUS estimates, $\hat{b}_{w, k}[t]$, for each media type $k$ within each workload $w$, (ii) confirming the feasibility of each workload's TTC value and selecting the appropriate service rate (i.e., selecting how many CUSs should be allocated to each workload's tasks), and (iii) devising and executing an algorithm to initialize or terminate CUs according to the demand volume. The first two items allow for microscale (i.e., local) control of Dithen, and they are discussed in parts 3 and 4 of this section, as well as in Sections III and IV. The last item allows for macroscale (i.e., global) control of the workload execution within Dithen; solutions for these aspects are analyzed in the first two parts of this subsection.

1. Task allocation and tracker operation via the GCI: To achieve each workload's TTC, the GCI divides the workload into chunks and sends these chunks to be processed by the spot instances of LCIs. Specifically, when a workload is submitted, the GCI examines the . \input folder to determine how many individually-executable tasks are present in the workload. Once this has been determined, the GCI executes a small number of the tasks in a "footprinting" stage. The goal of the "footprinting" stage is to determine: (i) an initial workload CUS estimate per input type; (ii) what chunk size to use (i.e., how many inputs to group together for execution by a single spot instance) such that the chunk processing time is comparable to the time interval between monitoring instances (described in Section II-D). Importantly, while the initial CUS estimate forms the basis for resource estimation in Dithen, it is often inaccurate because it is difficult to select a representative subset of the tasks when the execution time of these tasks is data dependent. For example, in many face detection [25] or transcoding workloads [32], the estimate that uses only "footprinting" data can be $50 \%$ higher than the final measured value because of the data dependency of these tasks. Another reason for such inaccuracy is that, when considering small subsets of tasks in some workloads (like Matlab-based applications), the CUS estimation is significantly offset by the disproportional amount of time needed to set up the execution environment (a.k.a. "deadband" time) in comparison to the actual code execution. Long deadband times in tasks mandate the grouping of several tasks into large chunks. Once the chunk size has been determined, the GCI connects to the LCIs via the XMLRPC protocol and instructs the LCI to execute the tasks in the chunk. The LCI writes entries to a MySQL database detailing the status of each task as it processes them, as well as execution time measurements once the task is completed. These are used in the Kalman estimation process of Subsection II-E-3. The GCI uses this database to determine which task should be placed in a chunk for an LCI in a manner analogous to a BitTorrent tracker [33]: the controller connects to the database to determine which tasks appear as: "pending", "processing" and "completed" and, based on the workload service rates (Section III), carries out the chunk allocation to available LCIs. This decoupling between the database writing by the LCIs and the database reading by the GCI prevents bottlenecks and minimizes the network traffic between the GCI and LCIs. A workload is marked as "completed" once the GCI detects that all tasks in the workload have been completed.

2. Spot instance initiation and termination via the GCI: A direct way to implement the scaling of the required instances is for the global controller instance to constantly match the total CUs billed in Dithen $\left[c_{\text {tot }}[t]\right.$ of (3)] to the total CUs required by all workloads $\left(\sum_{w=1}^{W[t]} r_{w}[t]\right)$ at each time instant $t$ by initializing or terminating spot instances (a.k.a. "reactive" control [34]). However, such an approach is not optimal for the following reasons: (i) $\sum_{w=1}^{W[t]} r_{w}[t]$ depends on the estimated CUSs required to complete the processing of each media type $k$ within each workload $w$; these estimations will not be accurate for all time instants and media types, and this will lead to unnecessary expenditure to initiate and pay for instances that may never be used due to estimation mismatch; (ii) due to the CU billing for large time intervals (e.g., Amazon EC2 spot instances are billed for one hour and GCE instances are billed in 10-minute slots), as well as the associated delay in initialization or termination of instances (in the order of minutes), rapid fluctuations in $\sum_{w=1}^{W[t]} r_{w}[t]$ (e.g., due to new workloads or workload cancellations by users) will cause bursts of initiation or termination requests and substantially increased "dead" time, which will be billed by the IaaS; (iii) without a control mechanism in place to absorb rapid fluctuations in demand, a flurry of spot instance requests may inadvertently cause unwanted spikes in spot instance pricing [1]. In the next two sections, we present our GCI proposal for best-effort TTC-abiding execution that ensures proportional fairness amongst all submitted workloads in Dithen.

3. Reliable CUS estimates for media types via Kalmanfilter realization: Due to the aforementioned inaccuracy of the CUS estimation based on the "footprinting" process, we propose the use of an adaptive CUS estimator that runs continuously during the execution of each workload. In our proposal, each LCI measures the average CUSs, $\tilde{b}_{w, k}$, required for each media type $k$ of each workload $w$ running on its instance types, by measuring the time to complete tasks between the previous and the current monitoring instance $(t-1$ and $t)$ and refining the measurement. We model this measurement operation mathematically by:

$$
\forall w, k, t: \tilde{b}_{w, k}[t]=\hat{b}_{w, k}[t]+v_{w, k}[t]
$$


where $v_{w, k}[t]$ is the measurement noise that deviates $\tilde{b}_{w, k}[t]$ from the ideal CUS estimate $\hat{b}_{w, k}[t]$ at time instant $t$. We assume that $v_{w, k}[t]$ can be modeled by independent, identically distributed (i.i.d.), zero-mean Gaussian random variables, i.e., $\forall w, k: \mathrm{V}_{w, k} \sim \mathcal{N}\left(0, \sigma_{v}^{2}\right)$.

We express the LCI estimation of the required CUSs for each workload and task type at time $t$ by:

$$
\forall w, k, t: \hat{b}_{w, k}[t]=\hat{b}_{w, k}[t-1]+z_{w, k}[t],
$$

with $z_{w, k}[t]$ the process noise [34], expressing variability in the execution time of each task type in each workload across time. We assume that $\forall w, k: z_{w, k}[t]$ can be modelled by i.i.d., zero-mean Gaussian random variables, i.e., $\forall w, k: \mathrm{Z}_{w, k} \sim$ $\mathcal{N}\left(0, \sigma_{z}^{2}\right)$. Given (4) and (5) and the fact that all noise terms are i.i.d., the noise variances are: $E\left\{\mathrm{~V}_{w, k}^{2}\right\}=\sigma_{v}^{2}, E\left\{\mathrm{Z}_{w, k}^{2}\right\}=\sigma_{z}^{2}$ and the noise covariance is $E\left\{\mathrm{~V}_{w, k} \mathrm{Z}_{w, k}\right\}=0$.

For the measurement and estimation model of (4) and (5), the optimal estimator for $\hat{b}_{w, k}[t]$ is known to be the Kalman filter [34], which provides for the following two time-update equations for our case $(\forall w, k, t)$ :

$$
\begin{gathered}
\pi_{w, k}^{-}[t]=\pi_{w, k}[t-1]+\sigma_{z}^{2}, \\
\kappa_{w, k}[t]=\frac{\pi_{w, k}^{-}[t]}{\pi_{w, k}^{-}[t]+\sigma_{v}^{2}},
\end{gathered}
$$

where $\pi^{-}$represents the initial update of the process covariance noise $\pi$, and $\kappa_{w, k}[t]$ is the Kalman gain of the $k$ th task type of the $w$ th workload at time instant $t$. Based on (6) and (7), the estimation of $\hat{b}_{w, k}[t]$ and the noise covariance update can be written as $(\forall w, k, t)$ :

$$
\hat{b}_{w, k}[t]=\hat{b}_{w, k}[t-1]+\kappa_{w, k}[t]\left(\tilde{b}_{w, k}[t-1]-\hat{b}_{w, k}[t-1]\right),
$$

$$
\pi_{w, k}[t]=\left(1-\kappa_{w, k}[t]\right) \pi_{w, k}^{-}[t] .
$$

Initialization of proposed CUS estimator per workload and task type: For $t=0$ and $\forall w, k$, the GCI initializes each Kalman-filter estimator with $\tilde{b}_{w, k}[0]$, established via the initial "footprinting" measurement per workload and input type, and sets: $\hat{b}_{w, k}[0]=\pi[0]=0$, and $\sigma_{z}^{2}=\sigma_{v}^{2}=0.5$.

GCI-based CUS estimation steps for each monitoring time instant $t, t \geq 1$ and $\forall w, k$ : (i) retrieve (via the ME) the CUS measurements per workload and task type to establish $\tilde{b}_{w, k}[t-$ 1]; (ii) perform the estimation of (6)-(9); (iii) retain the value of the estimated CUS per workload via (8) and (1).

4. TTC confirmation and service rate per workload: Let us assume that a reliable CUS estimation becomes available for workload $w, 1 \leq w \leq W\left[t_{\text {init }}\right]$, at monitoring time instant ${ }^{3}$ $t_{\text {init }}$. The GCI can then confirm that $d_{w}\left[t_{\text {init }}\right]$ (the requested TTC for workload $w$ at $t_{\text {init }}$ ) is achievable by Dithen under appropriate adjustment of the workload service rate, $s_{w}[t]$, for each monitoring time $t, t \geq t_{\text {init }}$. The service rate $s_{w}[t]$ corresponds to the number of CUs allocated to workload $w$

\footnotetext{
${ }^{3}$ The practical method to determine $t_{\text {init }}$ is described in Section V.
}

for the time interval between monitoring instants $t$ and $t+1$. Fractional values (e.g., $s_{w}[t]=0.7$ ) indicate that one $\mathrm{CU}$ is allocated to workload $w$ for $s_{w}[t] \times 100 \%$ of the time between $t$ and $t+1$. If the combination of $d_{w}\left[t_{\text {init }}\right]$ with the workload CUS estimate leads to $s_{w}\left[t_{\text {init }}\right]>N_{w, \max }$, with $N_{w, \max }$ a predetermined CU upper limit $\left(\forall w: N_{w, \max }=10\right.$ in our experiments), $d_{w}\left[t_{\text {init }}\right]$ is extended such that $s_{w}\left[t_{\text {init }}\right]=N_{w, \max }$. This process confirms $d_{w}\left[t_{\text {init }}\right]$ (or its extension) as the TTC for workload $w$.

The algorithm to determine $s_{w}[t]$ for each workload $w$ and each $t \geq t_{\text {init }}$ is presented in Section III and is carried out by the GCI based on the estimated CUS per workload. All LCIs of Dithen are given individual tasks from each workload $w$ according to $s_{w}[t]$ by the GCI.

\section{WORKLOAD EXECUTION With CONFIRMED TTC}

The GCI of Dithen ensures that each workload is executed within its remaining TTC by an allocation mechanism based on proportional fairness. The proportional fairness goal can then be stated as: at each monitoring instance $t$ and for each workload $w(1 \leq w \leq W[t])$, the GCI maximizes an objective function of the service rate, $s_{w}[t]$, that ensures all workloads are served proportionally to their CUS requirement, $r_{w}[t]$ [given by (1)], and inversely-proportionally to their TTC, $d_{w}[t]$. The latter is defined via an appropriate SLA mechanism once a workload is submitted for execution and an initial workload CUS estimate becomes available. In this work, we adopt the objective function:

$$
f\left(s_{w}[t]\right)=r_{w}[t] \ln \left(s_{w}[t]\right)-d_{w}[t] s_{w}[t] .
$$

The subtraction in (10) contrasts between the workload's CUS requirement, $r_{w}[t]$, and the TTC requirement, $d_{w}[t]$. In addition, following proportional fairness problems of other resource allocation work (notably in cellular network scheduling algorithms [35]), we opted for the use of the natural logarithm in the demand side of the objective function and pursue the maximization of $f\left(s_{w}[t]\right)$. Specifically, when the condition $\sum_{w=1}^{W[t]} r_{w}[t] \leq c_{\text {tot }}[t]$ is satisfied, it is straightforward to show that the optimal solution to the maximization of (10) is $\left(\forall s_{w}[t]>0\right)$

$$
s_{w}^{*}[t]=\arg \max \left\{f\left(s_{w}[t]\right)\right\}=\frac{r_{w}[t]}{d_{w}[t]} .
$$

This corresponds to the case where enough CUs are available to accommodate the demand and, therefore, allocation of service rates is carried out according to the required CUSs and TTC per workload at each monitoring time instant $t$. We can then calculate the total required CUs for optimal operation as:

$$
N_{\text {tot }}^{*}[t]=\sum_{w=1}^{W[t]} s_{w}^{*}[t]=\sum_{w=1}^{W[t]} \frac{r_{w}[t]}{d_{w}[t]} .
$$

However, due to volatility in both workload submission and CU availability in Dithen, it is likely that, for most monitoring instances $t, N_{\text {tot }}^{*}[t]$ differs from $N_{\text {tot }}[t]$ [the actual number of CUs, calculated by (2)]. In such cases, we can 
adjust the optimal service rates of (11) proportionally to the relative distance between $N_{\text {tot }}^{*}[t]$ and $N_{\text {tot }}[t]$. Specifically, if $N_{\text {tot }}^{*}[t]>N_{\text {tot }}[t]+\alpha$, with $\alpha$ the AIMD additive constant defined in the next section $(\alpha>0)$, we downscale the optimal service rate of each workload to:

$$
\begin{aligned}
\forall w: s_{w}^{-}[t] & =\frac{r_{w}[t]}{d_{w}[t]}\left(1-\frac{N_{\text {tot }}^{*}[t]-N_{\text {tot }}[t]-\alpha}{N_{\text {tot }}^{*}[t]}\right) \\
& =\frac{N_{\text {tot }}[t]+\alpha}{N_{\text {tot }}^{*}[t]} s_{w}^{*}[t] .
\end{aligned}
$$

If $N_{\text {tot }}^{*}[t]<\beta N_{\text {tot }}[t]$, with $\beta$ the AIMD scaling constant defined in the next section $(0<\beta<1)$, we upscale the optimal service rate of each workload to:

$$
\begin{aligned}
\forall w: s_{w}^{+}[t] & =\frac{r_{w}[t]}{d_{w}[t]}\left(1+\frac{\beta N_{\text {tot }}[t]-N_{\text {tot }}^{*}[t]}{N_{\text {tot }}^{*}[t]}\right) \\
& =\frac{\beta N_{\text {tot }}[t]}{N_{\text {tot }}^{*}[t]} s_{w}^{*}[t] .
\end{aligned}
$$

Finally, if $\beta N_{\text {tot }}[t] \leq N_{\text {tot }}^{*}[t] \leq N_{\text {tot }}[t]+\alpha$, the service rates of (11) are used. The use of $\alpha$ and $\beta$ in (13) and (14) ensures the service rate adjustment is considering the possible additive increase or multiplicative decrease that may occur via the AIMD algorithm after the service rate allocation is established for the interval between $t$ and $t+1$.

\section{Scaling With AdDitive Increase Multiplicative DECREASE}

For any CaaS system, $N_{\text {tot }}^{*}[t]$ of (12) and $N_{\text {tot }}[t]$ of (2) must be tightly coupled in order to ensure that the available compute-unit time can meet the service demand and TTC requirements at any instant. This is because, if $N_{\text {tot }}^{*}[t]$ is substantially higher than $N_{\text {tot }}[t]$, the delay to complete pending workloads can increase significantly and workload TTCs may be violated. Conversely, when $N_{\text {tot }}^{*}[t]$ is significantly smaller than $N_{\text {tot }}[t]$, several CUs may billed on the service unnecessarily. Therefore, and in conjunction with the fact that billing comes in hourly increments in Amazon EC2 spot-instances, sudden surges or dips demand will have a detrimental effect in the delay or cost of the deployment of Dithen. Hence, the goal of GCI component of Dithen is to maintain the resource reservation and workload service rates at the correct level. To this end, we propose the AIMD algorithm of Fig. 4. By controlling the additive and scaling constants, $\alpha$ and $\beta$ respectively, we can examine the behavior of Dithen under a wide variety of workload submissions. It should be noted that the corresponding problem of selecting which spot instances to terminate in the event that $N_{\text {tot }}[t]>N_{\text {tot }}^{*}[t]$ is trivial: per instance type, the prudent action is always to terminate spot instances with the smallest remaining time before renewal.

We refer to the work of Shorten et. al. [24] for details on the the stability and convergence properties of AIMD algorithms. A key aspect from their analysis is that fast convergence to an equilibrium state is achieved if $\beta$ is small and smoother transitions are expected if $\beta$ is close to unity [24]. After extensive experimentation, we opted for the values of $\beta=0.9$
$1 \%$ algorithm iterations for any monitoring time instant $t$

2 if $N_{\text {tot }}[t] \leq N_{\text {tot }}^{*}[t]$

3 incr $=$ TRUE

4 else

5 incr $=$ FALSE

$6 \%$ application of AIMD to tune $N_{\text {tot }}$ for the next instant

7 if incr $==$ TRUE

$8 N_{\text {tot }}[t+1]=\min \left\{N_{\text {tot }}[t]+\alpha, N_{\max }\right\} \%$ add more CUs 9 else

$10 N_{\text {tot }}[t+1]=\max \left\{\beta N_{\text {tot }}[t], N_{\text {min }}\right\} \%$ remove CUs

Fig. 4. Proposed AIMD algorithm; $\alpha$ is a positive constant, $\beta$ is a constant such that $0<\beta \leq 1$, and $N_{\max }$ and $N_{\min }$ are the upper and lower bounds for $N_{\text {tot }}[t]$.

and $\alpha=5$, which exhibit sufficiently-fast convergence while at the same time ensuring that CUs are not released prematurely.

While the AIMD algorithm tunes the total CU value, $N_{\text {tot }}[t]$, it does not select which instance types to deploy out of the $I$ possible. As detailed in Appendix A, the recent status of Amazon spot instance pricing provides for proportional increase of pricing according to the number of compute units per instance. Moreover, the single-CU instance type exhibits the minimum price volatility, thereby making it the safest instance type to use. Therefore, we opt to use only single$\mathrm{CU}$ instances in our experiments, i.e., $I=1$ and $p_{1}=1$, which alleviates the problem of selecting amongst a variety of instance types. However, depending on the evolution of pricing data from the IaaS provider, future work will expand our results into a variety of instance types.

\section{EXPERIMENTS}

In order to examine our proposals, we have deployed Dithen using single-CU m3 . medium spot instances of Amazon EC2 (see Appendix A for more details). As discussed in Section II, each instance has a corresponding LCI that is given new tasks to process once the GCI detects that it is idle. In addition, one reserved EC2 instance, serving as the GCI, calculates the Kalman filter estimates based on the CUS measurements per task. Under predetermined TTC per workload (which is confirmed by Dithen after an initial CUS estimation becomes available for the workload), it then derives the service rate per workload in fixed time periods (i.e., within 1-5 minute intervals), as described in Section III. This is communicated to the ME and the LCIs (see Fig. 1). The GCI also carries out the AIMD algorithm of Section IV in order to control the increase or decrease of spot instances according to the demand. The utilized AIMD parameters for all experiments were set to: $\alpha=5, \beta=0.9, N_{\min }=10, N_{\max }=100$ and $\forall w: N_{w, \max }=10$ (maximum service rate per workload). A SQL database is used by the ME to keep track of the tasks completed per workload. Finally, the produced results, as well as a summary of the intermediate progress, is communicated to the user by the web interface of the FE (Fig. 3). 


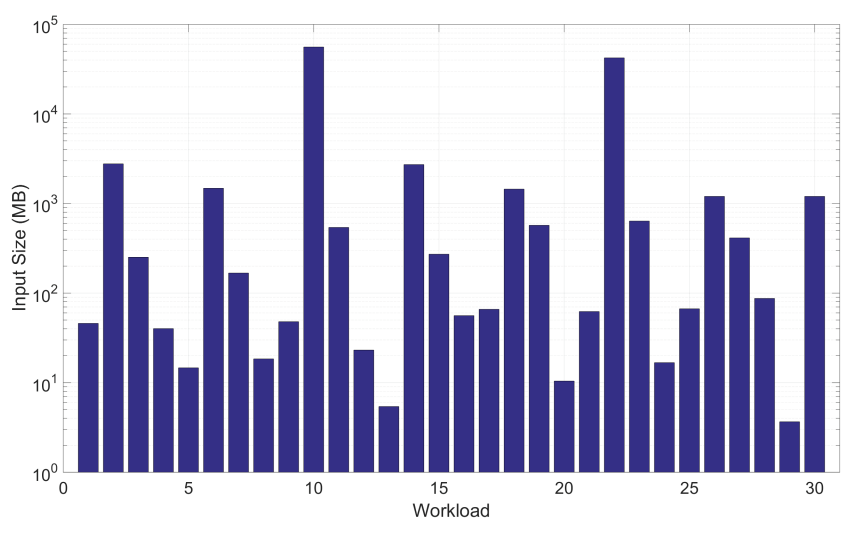

Fig. 5. Size of inputs for each of the thirty workloads used in our experiments.

\section{A. Utilized Workloads}

All multimedia inputs, processing scripts and executable files are placed on Amazon S3 via the uploading service ("add" button) available within the FE of Dithen [Fig. 3(a)]. Thirty different workloads, each with a random number of tasks were used in our experiments. Eight of the workloads were scripts running the Viola-Jones classifier [25] for face detection in images. The range of possible values for the number of inputs (i.e., images or videos) for these workloads was between 1 and 1000. Eight of the workloads were scripts using FFMPEG to transcode videos to different bitrates via a variety of codecs [32]. Each workload had between 1 and 20 videos to transcode, and we also added two large transcoding workloads with 200 and 300 videos. These were used to examine the responsiveness of the Dithen system under sudden spikes of demand. Seven of the workloads were using the OpenCV BRISK keypoint detector and descriptor extractor [36]. Finally, seven workloads used the Scale Invariant Feature Transform (SIFT) salient point descriptor [27], which was deployed as compiled Matlab code with the Mathworks deploytool. The total size of the inputs per workload is given in Fig. 5. Workloads were introduced once every five minutes in the order depicted in Figure 5.

\section{B. Performance of Kalman-based CUS Estimation}

The proposed Kalman-based CUS estimation process of Section III is compared against the "ad-hoc" estimator that carries out the CUS estimation of (8), albeit with the scaling coefficient being set to the fixed value: $\kappa_{w, k}[t]=0.1$, which was shown to perform best amongst other settings. Moreover, as an external comparison, we also utilize the well-known second-order autoregressive moving average (ARMA) estimator of Roy et. al. [37] that has been shown to perform well for workload forecasting. ARMA estimates the CUS required to complete a workload at time $t+1$ via

$$
\begin{aligned}
\hat{b}_{w, k}[t+1] & =\delta \times b_{\text {norm }, w, k}[t]+\gamma \times b_{\text {norm }, w, k}[t-1] \\
& +(1-\delta-\gamma) \times b_{\text {norm }, w, k}[t-2],
\end{aligned}
$$

where: $b_{\text {norm }, w, k}[t, t-1, t-2]$ are calculated by summing the total execution time of media type $k$ of workload $w$ at times $t, t-1, t-2$ and dividing it by the percentage of the workload that has been completed until then; and $\delta$ and $\gamma$ are scalars having the values recommended by Roy et. al. [37]. We chose ARMA as the most suitable benchmark because other workload forecasting methods (like the ARIMA model [38], [39]) require extensive past measurements from previous executions of other workloads, as well as a long sequence of measurements in order to produce reliable estimates, thereby making them unsuitable for our case.

Two representative examples of the convergence behaviors of all methods under comparison are given in Fig. 6 and Fig. 7. As illustrated in the figures, the Kalman and ad-hoc estimator exhibit an underdamped behavior until convergence. We can therefore use the slope of the CUS estimation across time to determine the monitoring time instant $t_{\text {init }}$ when the proposed Kalman and the ad-hoc estimator can provide a reliable CUS estimation per workload and task type. Specifically, when the slope of the CUS estimation becomes negative for the first time, each estimator establishes a CUS estimate for each workload with acceptable accuracy. However, ARMA does not exhibit such underdamped behavior, since it is a moving-average based estimator. Therefore, we relied on a conventional convergence detection criterion for ARMA: when the ARMA estimate deviation within the window of the last three measurements is found not to exceed $20 \%$ from the mean value derived from the values of the window (ten measurements are used for the case of 1-min monitoring), we determine that the estimate is reliable enough to be used. The setup for the window size and variability threshold was selected after testing with a variety of possible values. In the examples of Fig. 6 and Fig. 7, the time instant when each method reaches its reliable estimate under the described setup is marked with the red dotted vertical lines.

Table II presents the average time each estimator took to reach its CUS estimate for each workload type, as well as the CUS percentile mean absolute error (MAE). The summary over all workloads (per monitoring interval) is given at the bottom of the table. Evidently, the proposed Kalman-based approach reduces the average time to reach a reliable estimate by more than $20 \%$ in comparison to the other estimators and is found to be the quickest estimator in all but one case. At the same time, the proposed estimator attains comparable accuracy to the ad-hoc estimator and is found to be significantly superior to ARMA. This is especially pronounced in the case of the 1-minute monitoring, where the use of the proposed Kalmanbased approach instead of an ARMA approach provides for $38 \%$ reduction in estimation time and decreases the average estimation error from $16.4 \%$ to $4.5 \%$. This indicates that, under the usage of the proposed CUS estimator and 1-minute monitoring, the GCI is expected to have reliable estimates per workload (and thereby confirm that its requested TTC is achievable) within 6-11 minutes from its launch. Finally, when we compare the performance of one-minute monitoring to fiveminute monitoring, Table II shows that increase in the measurement granularity results in significant improvement in both the accuracy and time required to reach a reliable estimate. Specifically, for the proposed Kalman estimator, increased monitoring frequency reduces the the average estimation time 


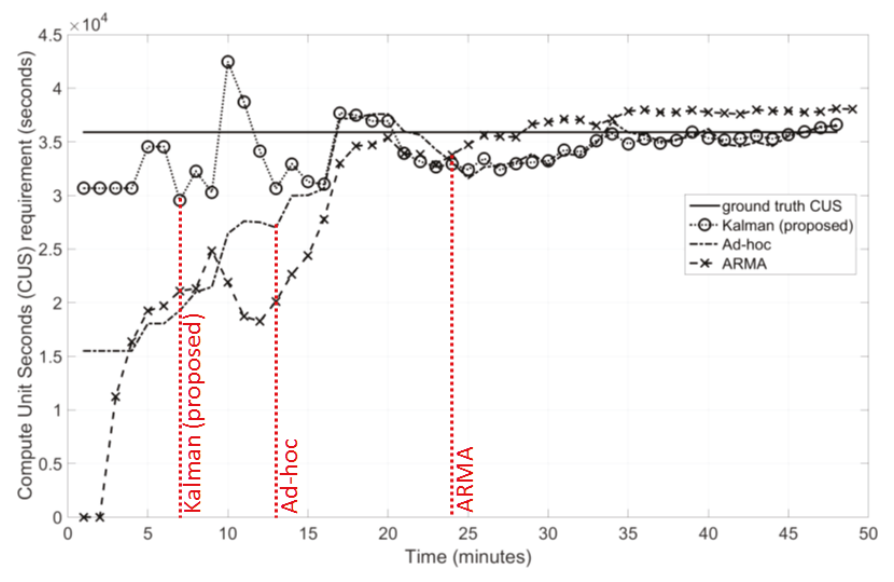

Fig. 6. Example of the convergence of various CUS estimation methods for the case of an FFMPEG workload under 1-min monitoring interval.

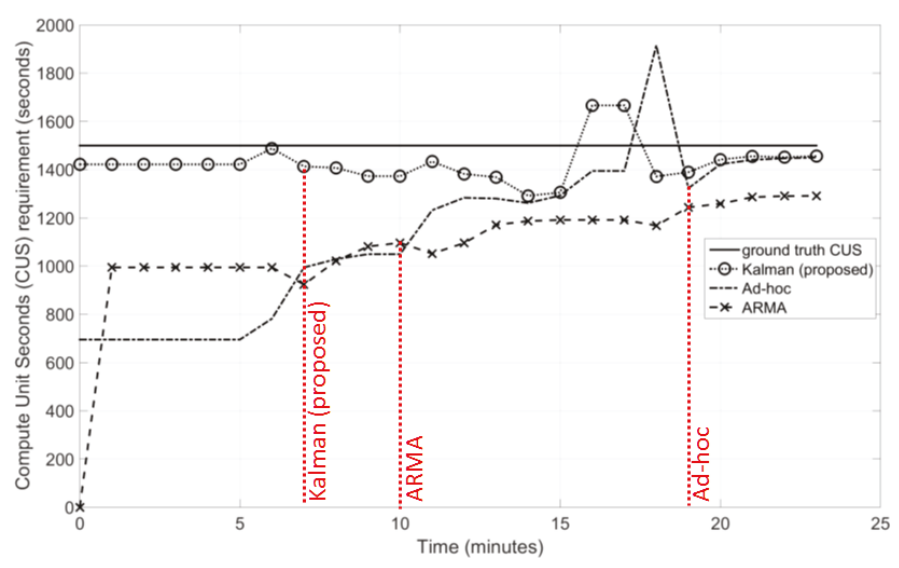

Fig. 7. Example of the convergence of various CUS estimation methods for the case of a SIFT descriptor (Matlab-based) workload under 1-min monitoring interval.

by $44 \%$ and reduces the overall MAE from $13.1 \%$ to $4.5 \%$.

\section{Results for Cumulative Cost of Workload Execution}

We now investigate the management of spot instances so that each workload is completed under a fixed TTC that is sufficiently large to allow for fluctuation in the number of utilized instances.

As external comparisons, our first choice is Amazon's Autoscale service (termed as "Amazon AS"), which is widely deployed in practice [40]. Amazon AS does not carry out CUS estimation or TTC-abiding execution, and one can only control the number of instances based on CPU utilization and bandwidth constraints. Therefore, under these conditions, we configured all workloads to execute within an Amazon AS group that examines the average CPU usage at all utilized CUs in five-minute intervals. If the group detected that the average CPU utilization was more than $20 \%$, new instances were started ${ }^{4}$. Otherwise, Amazon AS terminated some of the active instances. We then executed all workloads in Amazon AS and measured the longest time to complete a workload under two scaling policies. The first represented a conservative approach where reducing the execution time is not of critical importance. In this case, a single instance is added or removed when a monitoring interval occurs. The longest completion time was found to be $2 \mathrm{hr} 7 \mathrm{~min}$. The second scaling policy started and stopped ten instances instead of one, to represent a scenario where reduced execution time is of importance. In this case, the longest time to complete a workload was found to be $1 \mathrm{hr}$ and $37 \mathrm{~min}$. Both of these times were then used as the two fixed TTC settings for all workloads in Dithen.

Beyond Amazon AS, in order to benchmark our AIMDbased scaling of Fig. 4 against other alternatives for CU adjustment, we utilized the mean-weighted-average and linearregression methods of Gandhi, Krioukov et. al. [17], [41] (termed "MWA" and "LR", respectively) to set the number of CUs for the next monitoring interval, $N_{\text {tot }}[t+1]$. We selected MWA and LR for our comparisons because previous work [17] has shown them to be amongst the most accurate predictive resource controllers. Both MWA and LR utilized the proposed Kalman-based CUS estimation process and the service rate allocation of (12) to determine when to increase or decrease CUs. Specifically: (i) MWA sets the number of CUs via

$$
N_{\text {tot }}[t+1]=\frac{1}{6} \sum_{i=t-5}^{t} N_{\text {tot }}^{*}[i],
$$

where $N_{\text {tot }}^{*}$ is the optimal number of CUs derived via (12) for each monitoring time instant; (ii) LR sets $N_{\text {tot }}[t+1]$ to be the result of extrapolating the line derived via linear regression from $\left\{N_{\text {tot }}^{*}[t], \ldots, N_{\text {tot }}^{*}[t-5]\right\}$ (current plus five previous CU settings). Finally, in order to see the performance of the directcompensation approach, we also utilized the case where no filtering or other adjustment is being used and we simply set $N_{\text {tot }}[t+1]=N_{\text {tot }}^{*}$ (termed as "Reactive").

Figure 8 and Figure 9 show the cumulative cost of each approach during the course of both experiments with the two TTC values. Evidently, the cost of Amazon AS is significantly higher than that of all other approaches. This is primarily because the Amazon AS is the only approach that does not use CUS estimations and instead bases its decisions solely on CPU utilization. Therefore, it continues to scale up the number of instances even when it is nearing completion of the workloads' processing and only scales down after workloads have been completed and CPU utilization decreases due to inactivity.

Amongst MWA, LR and Reactive, MWA is superior as it incurs less cost for the majority of the experiment (and, as expected, Reactive is the worst). However, all three methods end up incurring very comparable cost for the completion of all workloads. Interestingly, Reactive turns out to be (marginally) the cheapest of the three for this experiment even though it

\footnotetext{
${ }^{4}$ After extensive experimentation, the value of $20 \%$ was found to provide for the best results with Amazon AS. This is because average utilization values between $18 \%$ and $22 \%$ represent the average CPU usage observed within active time intervals when an instance alternates between downloading files (2\%-10\% CPU utilization) and actually executing a compute-intensive task (close to $100 \%$ CPU utilization).
} 
TABLE II

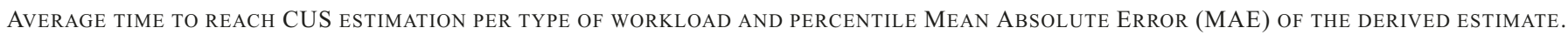
THE LAST COLUMN PRESENTS THE PERCENTILE TIME REDUCTION WHEN SWITCHING FROM 5-MIN MONITORING TO 1-MIN MONITORING INTERVALS. THE BEST RESULT PER CATEGORY IS INDICATED IN BOLDFACE FONT.

\begin{tabular}{|c|c|c|c|c|c|}
\hline Control interval & \multicolumn{2}{|c|}{ 5-min monitoring } & \multicolumn{2}{|c|}{ 1-min monitoring } & \multirow{2}{*}{$\begin{array}{l}\text { Time Reduction }(\%) \text { by going } \\
\text { from } 5 \text {-min to } 1 \text {-min monitoring }\end{array}$} \\
\hline Face Detection & Time & MAE $(\%)$ & Time & MAE $(\%)$ & \\
\hline Kalman-based & $13 \mathrm{~m} \mathrm{45s}$ & 5.6 & $10 \mathrm{~m} \mathrm{38s}$ & 4.6 & 22.7 \\
\hline Ad-hoc & $28 \mathrm{~m} \mathrm{08s}$ & 4.5 & $17 \mathrm{~m} \mathrm{53s}$ & 5.3 & 36.4 \\
\hline ARMA & $23 \mathrm{~m} \mathrm{08s}$ & 22.1 & $12 \mathrm{~m} \mathrm{08s}$ & 27.8 & 47.6 \\
\hline Transcoding & Time & MAE (\%) & Time & MAE (\%) & \\
\hline Kalman-based & $16 \mathrm{~m} \mathrm{53s}$ & 14 & $07 \mathrm{~m} \mathrm{54s}$ & 7.8 & 53.2 \\
\hline Ad-hoc & $26 \mathrm{~m} 53 \mathrm{~s}$ & 8.9 & $10 \mathrm{~m} \mathrm{36s}$ & 1.5 & 60.6 \\
\hline ARMA & $28 \mathrm{~m} \mathrm{08s}$ & 13.9 & $18 \mathrm{~m} \mathrm{45s}$ & 18.1 & 33.4 \\
\hline Feat. Extraction & Time & MAE (\%) & Time & MAE (\%) & \\
\hline Kalman-based & $13 \mathrm{~m} \mathrm{34s}$ & 12.1 & $11 \mathrm{~m} \mathrm{54s}$ & 1.4 & 12.3 \\
\hline Ad-hoc & $18 \mathrm{~m} \mathrm{34s}$ & 6.4 & $20 \mathrm{~m} 24 \mathrm{~s}$ & 1.9 & -9.9 \\
\hline ARMA & $20 \mathrm{~m} \mathrm{43s}$ & 5.7 & $11 \mathrm{~m} \mathrm{09s}$ & 12.1 & 46.2 \\
\hline SIFT & Time & MAE $(\%)$ & Time & MAE $(\%)$ & \\
\hline Kalman-based & $21 \mathrm{~m} \mathrm{26s}$ & 20.6 & $06 \mathrm{~m} \mathrm{18s}$ & 4.1 & 70.6 \\
\hline Ad-hoc & $23 \mathrm{~m} 54 \mathrm{~s}$ & 18.9 & $08 \mathrm{~m} \mathrm{06s}$ & 0.1 & 66.1 \\
\hline ARMA & $20 \mathrm{~m} \mathrm{00s}$ & 20.1 & $15 \mathrm{~m} \mathrm{00s}$ & 7.6 & 25.0 \\
\hline Overall Average & Time & MAE (\%) & Time & MAE (\%) & \\
\hline Kalman-based & $16 \mathrm{~m} \mathrm{25s}$ & 13.1 & $09 \mathrm{~m} \mathrm{11s}$ & 4.5 & 44.1 \\
\hline Ad-hoc & $24 \mathrm{~m} \mathrm{22s}$ & 9.7 & $14 \mathrm{~m} 15 \mathrm{~s}$ & 2.2 & 34.6 \\
\hline ARMA & $23 \mathrm{~m} \mathrm{00s}$ & 15.5 & $14 \mathrm{~m} 15 \mathrm{~s}$ & 16.4 & 38.0 \\
\hline
\end{tabular}

TABLE III

SUMMARY OF OVERALL COST OF DIFFERENT METHODS AS WELL AS COMPARISONS WITH THE PROPOSED METHOD AND THE LOWER BOUND

\begin{tabular}{|c|c|c|c|c|c|c|}
\hline System & AIMD (proposed) & Reactive & MWA & LR & $\mathrm{AS}$ & LB \\
\hline Overall cost $(\$)$ & 0.41 & 0.51 & 0.52 & 0.53 & 1.02 & 0.22 \\
\hline Average cost reduction of proposed vs. other methods $(\%)$ & - & 20 & 21 & 23 & 60 & - \\
\hline Average cost increase above LB $(\%)$ & 86 & 132 & 136 & 141 & 364 & - \\
\hline Max. \# of instances at any time by each method & 13 & 28 & 21 & 24 & 91 & - \\
\hline
\end{tabular}

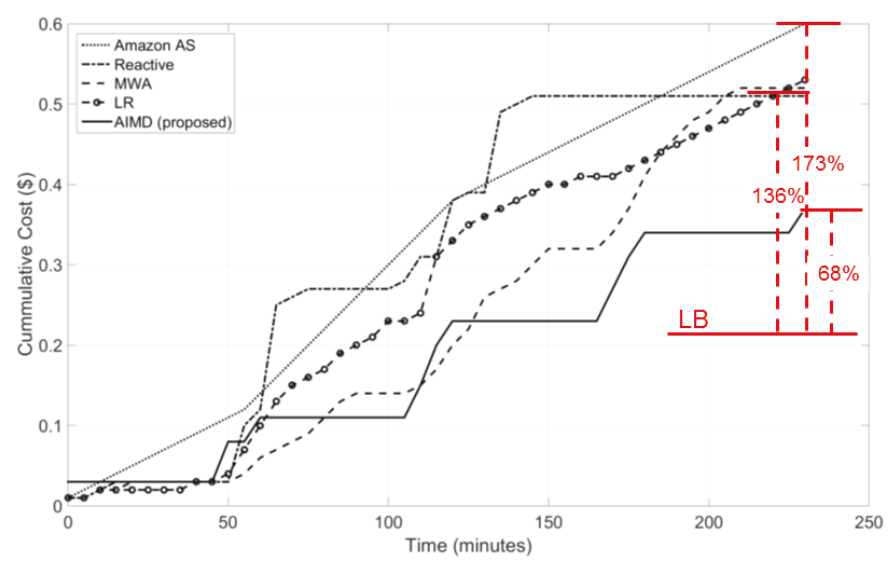

Fig. 8. Cumulative cost of processing all workloads of Fig. 5 under fixed TTC of $2 \mathrm{hr} 7 \mathrm{~min}$ per workload.

uses the largest number of instances of the three methods at one point. The reason for this is that, while Reactive scales up very quickly it also scales down rapidly and, for this particular experiment, this behaviour worked in its favour. However, this is not expected to be always the case, as Reactive does leave many instances idle for a large portion of their billed time.

The proposed AIMD-based scaling initially scales up when it detects the large workloads, then maintains this level, and then begins to scale down as it nears the experiment completion. For the experiments of Figure 8, this leads to overall savings of 30\% against MWA, 29\% against LR, 27\% against Reactive and $38 \%$ against Amazon AS. For the experiments of Figure 9, the equivalent savings were: $14 \%, 15 \%, 12 \%$ and $69 \% 0^{5}$. Overall, beyond the advantage of providing for scaledup execution under TTC constraints, the 38\%-69\% savings demonstrated in Figure 8 and Figure 9 allow for significant profit margin for cloud service providers that would deploy large-scale multimedia applications via the techniques used in Dithen, versus utilizing Amazon AS directly.

The overall savings for both experiments, as well as the maximum number of instances used by the proposed algorithm against all other benchmarks are summarized in Table III. It should be emphasized that, beyond the cost savings, all the workloads in the proposed AIMD approach finished before their execution time exceeded the predetermined TTC of

\footnotetext{
${ }^{5}$ It should be noted that the controller does incur some overhead cost. If we were to subtract the cost from Amazon AS (Reactive, MWA and LR also require a controller and thus have the same overhead as AIMD) it would not improve its performance by no more than $5 \%$, with this percentage diminishing as the workload size increases. Finally, it is also important to note that the controller instance does not have to run in AWS; instead, it could operate under a captive computing environment, thereby incurring no billing cost from the cloud provider.
} 


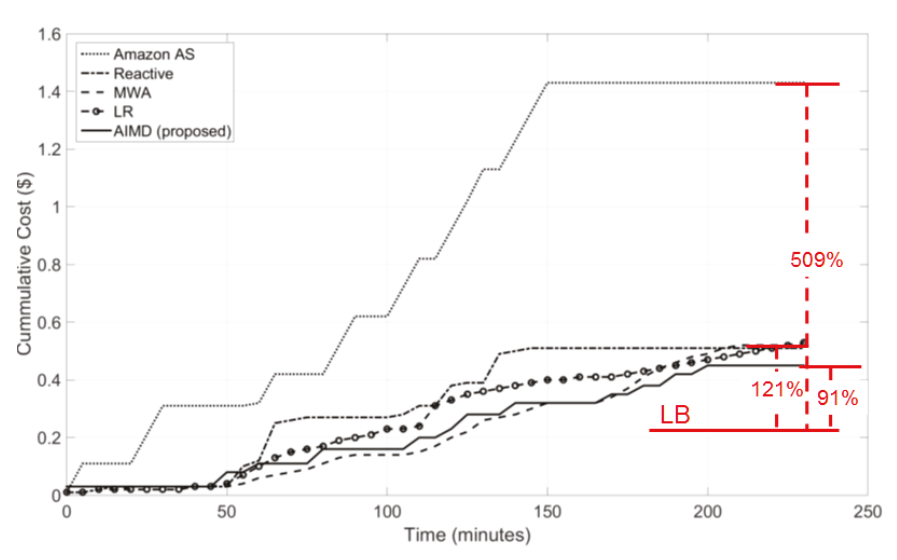

Fig. 9. Cumulative cost of processing all workloads of Fig. 5 under fixed TTC of $1 \mathrm{hr} 37 \mathrm{~min}$ per workload.

each experiment. Such TTC-abiding execution is a significant feature that Amazon AS cannot provide.

Finally, the bottom right of Figure 8 and Figure 9 includes a red horizontal line indicating the estimated billing if all workloads would be processed such that all billed instances would be occupied $100 \%$ of the time. This constitutes the lower bound for the billing cost (termed "LB") as no operational approach can achieve lower cost. Evidently, the proposed approach incurs $68 \%-91 \%$ higher cost than LB, but all other approaches incur $135 \%-510 \%$ higher cost than LB. It should be noted that both the LB and all the examined approaches include the delay to transport of data to and from the instances. If this would be removed, all costs would be lowered by approximately $27 \%$. Overall, the results of Figure 8 and Figure 9 demonstrate that the proposed AIMD-based scaling of CUs is a simple and effective method towards approaching the lowest possible cost incurred from the cloud computing infrastructure, while at the same time satisfying the TTC constraint of each workload.

\section{Comparison Against Amazon Lambda}

Recently, Amazon begun offering its own CaaS service for the execution of Javascript code via its Lambda service. Despite this being more limiting due to the inefficiency of Javascript code, we compared the cost of running three large Javascript-based workloads on Dithen and Lambda. In this experiment we ran "blur", "rotate" and "convolve" operations from the Javascript version of the widely-used ImageMagick image manipulation program [26]. We chose these functions as they represent a cross section of computational requirements of the various ImageMagick functions. Each function was executed on 25,000 images encompassing a wide variety of sizes and pixel counts. We also opted for the 1024MB-memory configuration for all Lambda functions to avoid any memory bottlenecks during execution. Again, Dithen was tuned to match the execution time of each workload in Lambda. This was done because the latter is dependent on how quickly requests can be sent to call the functions through the Amazon Web Service Command Line interface (or any other such API),
TABLE IV

Average Cost of IMAGEMAGICK FUnCTIONS PER IMAGE OF THE 25,000 DATASET FOR DITHEN AND AMAZON'S LAMBDA.

\begin{tabular}{|c|c|c|c|}
\hline Function & Lambda Cost (\$) & Dithen Cost (\$) & Ratio \\
\hline Blur & $4.74 \times 10^{-5}$ & $1.42 \times 10^{-5}$ & 3.34 \\
\hline Convolve & $1.68 \times 10^{-5}$ & $6.05 \times 10^{-6}$ & 2.78 \\
\hline Rotate & $5.5 \times 10^{-6}$ & $6.8 \times 10^{-6}$ & 0.81 \\
\hline Overall Average & $2.32 \times 10^{-5}$ & $9.20 \times 10^{-6}$ & 2.52 \\
\hline
\end{tabular}

while the execution time for workloads in Dithen is completely tunable based on their specified TTC. This flexibility of TTC-abiding execution per workload is an advantage of our proposal against Lambda.

A comparison of the cost of executing the workloads is given in Table IV. It is interesting to notice that, as the run time of the function decreases, Lambda becomes a more viable option. For example, the average cost of running the most compute-intensive function (Blur function in Table IV) was 3.34 times higher on Lambda than it was on Dithen. In contrast the average cost of running the fastest and least compute-intensive function (the rotate function) was found to be slightly less on Lambda than on Dithen. This result can be understood as follows. AWS Lambda allocates cores based on the memory consumption. For example, if the Lambda functions run on an EC2 instance with 4 GB memory and 2 cores and the functions require only $1 \mathrm{~GB}$ of memory, Lambda will allocate only $\frac{1}{4} \times 2$ cores, so it won't utilize the full processing power of the instance, thereby making the functions run longer. This implies that, when Lambda handles low-load tasks (i.e., easily executable even when a non-dedicated core is available), it becomes advantageous to Dithen. However, when high-load tasks are executed, the pricing and core allocation of Lambda becomes less advantageous since the execution time of complex tasks is significantly prolonged in comparison to Dithen (which always allocates an entire core per task, regardless of the task's complexity). Therefore, beyond simple web front-end type of tasks (which is the ideal application domain for AWS Lambda-hence its design and pricing being built around this), Lambda is not advantageous for the vast majority of more advanced computing tasks handled by a more generic and extendable CaaS platform like Dithen. Overall, we were able to run the workloads on Dithen at more than 2.5 times lower cost (60\% reduction) in comparison to Amazon Lambda. This provides for substantial profit margin for a cloud service provider to deploy a large-scale multimedia application via the proposed approach instead of Lambda.

\section{E. Deep Learning and Split-Merge Workloads}

We conclude our experiments by examining the performance of our platform when processing more complicated workloads, namely: (i) an image classification application based on a group of deep convolutional neural networks (CNN) [42] that have been trained on ImageNet [43] and (ii) a large-scale word histogram calculation, which is the standard example used with MapReduce-type of processing [28].

The first example is a representative case for a Split-Merge workload since multiple deep $\mathrm{CNNs}$ are used to classify 


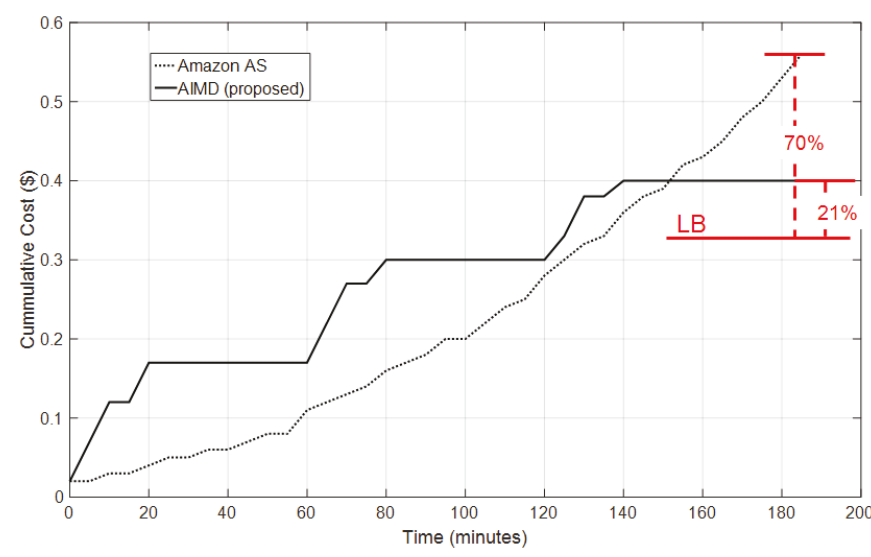

Fig. 10. Cumulative cost of an image classification workload based on deep CNNs.

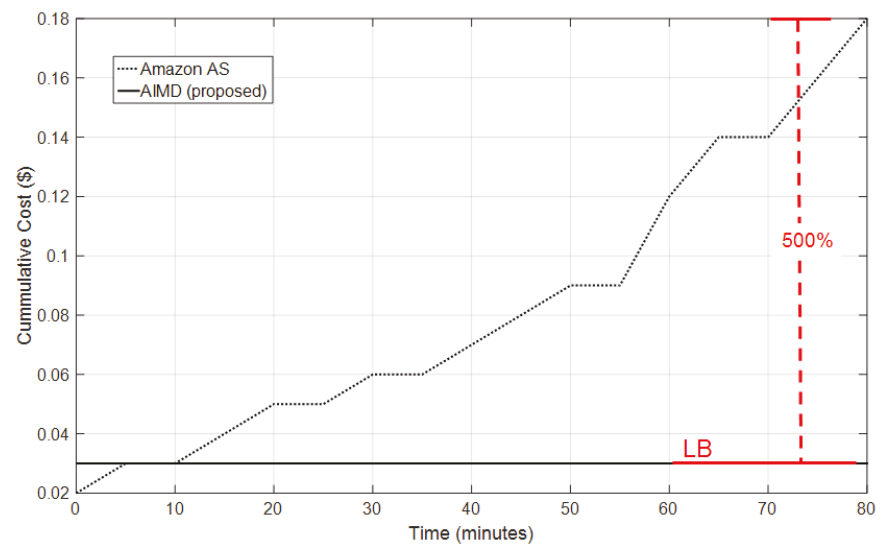

Fig. 11. Cumulative cost of a word histogram calculation workload based on Split-Merge (the workload is the standard one used within MapReduce testing [28])

each input image during the split stage and their results are aggregated via a voting process in the merge stage in order to produce the final classification result per image [43]. The inputs used for this workload comprised all images of the "Holidays" dataset [30], as well as 50,000 additional images from ImageNet.

In the second example, the workload measured the number of occurrences of words in a text file and then this was aggregated into a word histogram by a separate Reduce (or merge) instance. This is similar to a number of text based workloads where text data is analysed in order to gain insights into trends in market sentiment. The inputs used to test this workload were a selection of the Project Gutenberg [44] library which was approximately 14,000 text files and 5.5GB of data. This example is used in order to demonstrate that, while Dithen is more amenable to multimedia workloads (where the partitioning is inherent), it can also be used for more general workloads such as market sentiment analysis and the semantic analysis of text. It should be noted that, beyond testing, Dithen could be used for deep learning training workloads as well. For example Tensorflow could be used with batches of training sets and the results of such batch- based training could be merged at a later stage, after several iterations have been carried out in batch mode. We plan to report on such experiments in a future paper.

The experiments were invoked via the front end following the process described in Section II-B and experimental benchmarking of the incurred cost occurred as described in Section V-C. Specifically, the workloads were first executed using Amazon's Autoscaling service (commonly used for such systems [45]), which was used to determine the TTC to use for our platform ${ }^{6}$ (since Amazon AS does not allow for TTCabiding execution). Based on this process, the TTC was set to $1 \mathrm{hr} 35 \mathrm{~min}$ for the first example and $1 \mathrm{hr} 05 \mathrm{~min}$ for the second example. In order to account for the time for the Merge step of each of the two workloads, the TTC for each Split stage was set to $90 \%$ of the overall TTC.

The cumulative cost of the image classification workload can be seen in Figure 10. Similar to previous examples, the cost of Amazon AS is 38\% higher than the cost of the AIMD approach of Dithen. We can also see that the cost of this workload in Dithen is only $21 \%$ higher than the lower bound, while the cost of the Amazon AS approach is 70\% higher than the lower bound.

The cumulative cost of the word histogram calculation workload is depicted in Figure 11. In this case, the cost of Amazon AS is six times that of the Dithen platform and the lower bound. Interestingly, in this case, the cost incurred by the AIMD approach of Dithen is extremely close to the lower bound (less than $\$ 0.005$ higher) and remains constant at 3 cents. This result is achieved because, in this particular case, Dithen was able to quickly and reliably identify the CUSs required to complete the Split tasks and determined that 3 spot instances suffice for the completion of the workload within the predetermined TTC, and below the 1 hour mark (at which point additional charges are levied by AWS). Therefore, it avoided the unnecessary launch of new instances and its cost remained constant at 3 cents since the Split-Merge workload execution finished in 55 minutes.

Overall, these examples show that the platform can be used to substantially lower the execution costs of complex workloads and, in certain circumstances, it is even possible for Dithen to approach the lower bound for the incurred cost.

\section{CONCLUSIONS}

We present Dithen, a novel Computation-as-a-Service framework, which supports the direct upload and execution of multimedia processing workloads. The Dithen architecture comprises multiple spot instances that execute tasks within the workloads until their compute units are fully utilized. Dithen uses the Additive Increase Multiplicative Decrease (AIMD) algorithm for the allocation or termination of compute units and a Kalman-based estimator for the required compute-unitseconds for each type of task with each workload. Experiments based on Amazon EC2 spot instances demonstrated

\footnotetext{
${ }^{6}$ For simplicity and brevity in our exposition, we did not include the results with the remaining methods in our presented comparisons (MWA, LR and Reactive), as they were found to incur similar overhead as in the previous experiments. Furthermore, no results are presented for Amazon Lambda, as Lambda cannot support such complex processing tasks.
} 


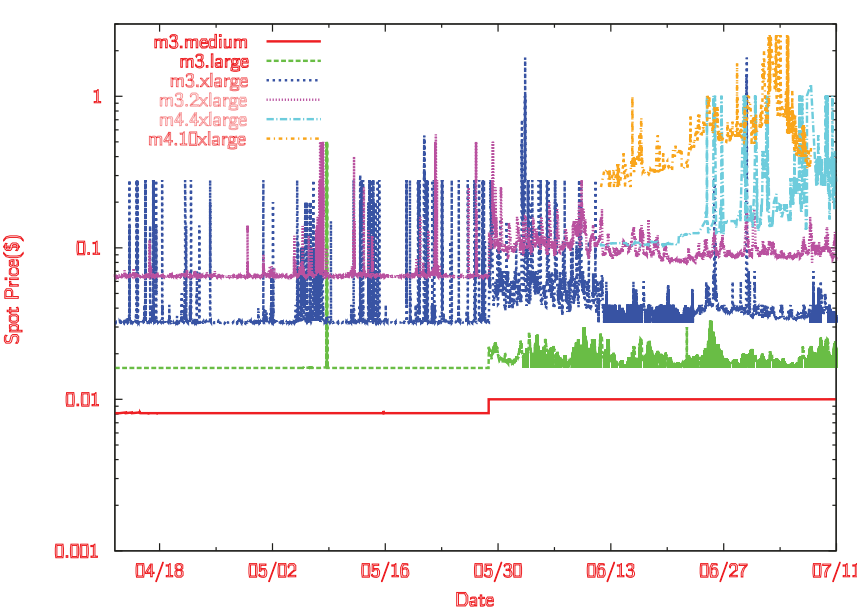

Fig. 12. Spot Price for various instance types from $11^{\text {th }}$ of April to the $11^{\text {th }}$ July 2015

that, unlike all existing Platform-as-a-Service and Softwareas-a-Service frameworks, Dithen provides for extreme scaling of commodity multimedia computing tasks (like large-scale transcoding, face detection and feature extraction workloads), without requiring any modification in the users' code base, and at substantially-reduced cost against all other alternatives. Moreover, unlike other services, Dithen allows for execution under time-to-completion constraints. The baseline form of the proposed service is freely available at www.dithen.com.

\section{APPENDIX A}

We briefly analyze the computation costs of Linux instances on AWS EC2, as EC2 is considered to be the largest public cloud service provider today [46] and our system is tested and deployed on the EC2 infrastructure. A comparison of the cost and EC2 compute units (ECUs) of various instance types is given in ${ }^{7}$ Table V. An ECU is defined as "equivalent CPU capacity of a 1.0-1.2 GHz 2007 Opteron or 2007 Xeon processor". The $\mathrm{m} 3$. medium instance (utilized in this paper) is a single $\mathrm{CU}$ instance with clock speed of $3.0-3.6 \mathrm{GHz}$. From the table we can also see that the larger instances consist of increasing numbers of CUs (i.e., virtual cores available for computations) with similar clock speeds. We can also see that the "On Demand" cost and spot prices are both linearlydependent on the number of CUs. Thus, we can conclude that it is more efficient to use a large number of cheaper instances than small number of more expensive instances, as it allows for greater granularity when controlling the number of active instances without any corresponding increase in cost.

From Table $\mathrm{V}$ we can also see the difference between the "On Demand" cost and the Spot price ${ }^{8}$. Spot instances are instances that will only function when a user's bid is greater than the current spot price. Essentially, the user gives up certainty of having computational resources available, in

\footnotetext{
${ }^{7}$ Table V does not include all instance types available on Amazon's EC2. However, all non-included instances are memory, computation or storage variants of the instances depicted in Table $\mathrm{V}$.

${ }^{8}$ The Spot prices depicted in Table V were taken on the $10^{\text {th }}$ July 2015.
}

exchange for a significant reduction in the cost. We can see from Table $\mathrm{V}$ that this reduction ranges from $78 \%$ to $89 \%$. However, it is difficult to run a CaaS service without guarantees of the availability of computational resources, so an analysis of the fluctuation of the spot price is necessary to determine if spot instances should be utilized.

The spot instance price for various instance types in the three-month period from the $11^{\text {th }}$ of April to the $11^{\text {th }}$ July 2015 is shown in ${ }^{9}$ Figure 12. Evidently, the volatility of the spot price is proportional to number of CUs that an instance possesses. Therefore, while it would be difficult to rely on a $4.10 x$ large spot instance, the spot price of the m3 . medium spot instance is remarkably stable. Specifically, at no point in the three month period does the $\mathrm{m} 3$. medium spot price exceed $\$ 0.01$. Therefore, we can conclude that a significant reduction in cost can be achieved by using m3. medium spot instance with little effect on the reliability of the service, and with more flexibility than when using larger spot instances with more CUs.

\section{REFERENCES}

[1] Y. Song, M. Zafer, and K.-W. Lee, "Optimal bidding in spot instance market," in Proc. IEEE INFOCOM, 2012, pp. 190-198.

[2] L. Zhang, Z. Li, and C. Wu, "Dynamic resource provisioning in cloud computing: A randomized auction approach," in Proc. of IEEE INFOCOM, 2014

[3] X. Nan, Y. He, and L. Guan, "Optimal allocation of virtual machines for cloud-based multimedia applications," in IEEE Int. Conf. Multimedia Signal Proc. (MMSP). IEEE, 2012, pp. 175-180.

[4] T. Hobfeld, R. Schatz, M. Varela, and C. Timmerer, "Challenges of QoE management for cloud applications," IEEE Comm. Mag., vol. 50, no. 4, pp. 28-36, 2012.

[5] S. Islam and J.-C. Grégoire, "Giving users an edge: A flexible cloud model and its application for multimedia," Fut. Gen. Comp. Syst., vol. 28, no. 6, pp. 823-832, 2012.

[6] Y. Andreopoulos and M. van der Schaar, "Incremental refinement of computation for the discrete wavelet transform," IEEE Trans. on Signal Process., vol. 56, no. 1, pp. 140-157, 2008.

[7] V. Spiliotopoulos et al., "Quantization effect on vlsi implementations for the 9/7 dwt filters," in Proc. IEEE Int. Conf. on Acoust., Speech, and Signal Process., ICASSP'01. IEEE, 2001, vol. 2, pp. 1197-1200.

[8] Y. Andreopoulos et al., "A local wavelet transform implementation versus an optimal row-column algorithm for the $2 \mathrm{~d}$ multilevel decomposition," in Proc. IEEE Int. Conf. on Image Process., ICIP 2001. IEEE, 2001, vol. 3, pp. 330-333.

[9] Y Andreopoulos et al., "A new method for complete-to-overcomplete discrete wavelet transforms," in Proc. 14th IEEE Int. Conf. on Digital Signal Process., DSP 2002. IEEE, 2002, vol. 2, pp. 501-504.

[10] N. Kontorinis et al., "Statistical framework for video decoding complexity modeling and prediction," IEEE Trans. on Circ. and Syst. for Video Technol., vol. 19, no. 7, pp. 1000-1013, 2009.

[11] K. Masiyev et al., "Cloud computing for business," in IEEE Int. Conf. Appl. of Inf. and Comm. Tech. (AICT). IEEE, 2012, pp. 1-4.

[12] I Andreopoulos et al., "A hybrid image compression algorithm based on fractal coding and wavelet transform," in Proc. IEEE Int. Symp. Circuits and Systems, 2000 (ISCAS 2000). IEEE, 2000, vol. 3, pp. 37-40.

[13] Y. Andreopoulos et al., "High-level cache modeling for 2-d discrete wavelet transform implementations," Journal of VLSI signal processing systems for signal, image and video technology, vol. 34, no. 3, pp. 209226, 2003.

[14] M. Schwarzkopf, A. Konwinski, M. Abd-El-Malek, and J. Wilkes, "Omega: Flexible, scalable schedulers for large compute clusters," in Proceedings of the 8th ACM European Conference on Computer Systems, 2013, EuroSys '13, pp. 351-364.

\footnotetext{
${ }^{9}$ In the case of $\mathrm{m} 4.4 \mathrm{x}$ large and $\mathrm{m} 4.10 \mathrm{xlarge}$ data was only available from the $11^{\text {th }}$ June.
} 
TABLE V

Cost of Various Linux Instances on the Amazon EC2 Platform in the North Virginia Region

\begin{tabular}{|l|c|c|c|c|c|c|}
\hline Instance Type & m3.medium & m3.large & m3.xlarge & m3.2xlarge & m4.4xlarge & m4.10xlarge \\
\hline \hline EC2 compute units (ECUs) & 3 & 6.5 & 13 & 26 & 53.5 & 124.5 \\
\hline CUs & 1 & 2 & 4 & 8 & 16 & 40 \\
\hline On-demand cost (\$) & 0.067 & 0.133 & 0.266 & 0.532 & 1.008 & 2.52 \\
\hline Spot price (\$) & 0.0081 & 0.0173 & 0.0333 & 0.066 & 0.1097 & 0.5655 \\
\hline Cost reduction when using spot (\%) & 88 & 87 & 87 & 88 & 89 & 78 \\
\hline
\end{tabular}

[15] E. Boutin, J. Ekanayake, W. Lin, B. Shi, J. Zhou, Z. Qian, M. Wu, and L. Zhou, "Apollo: scalable and coordinated scheduling for cloud-scale computing," in Proc. USENIX Symp. on Operating Systems Design and Implementation (OSDI), 2014

[16] K. Ousterhout, P. Wendell, M. Zaharia, and I. Stoica, "Sparrow: distributed, low latency scheduling," in Proceedings of the TwentyFourth ACM Symposium on Operating Systems Principles, 2013, pp. 69-84.

[17] A. Gandhi, M. Harchol-Balter, R. Raghunathan, and M. A. Kozuch, "Autoscale: Dynamic, robust capacity management for multi-tier data centers," ACM Trans. Comput. Syst., vol. 30, no. 4, pp. 14:1-14:26, Nov. 2012.

[18] A. Paya and D. Marinescu, "Energy-aware load balancing and application scaling for the cloud ecosystem," IEEE Trans. on Cloud Comp., vol. PP, no. 99, pp. 1-1, 2015.

[19] R. Ranjan, K. Mitra, and D. Georgakopoulos, "Mediawise cloud content orchestrator," J. of Int. Serv. and Appl., vol. 4, no. 1, pp. 1-14, 2013.

[20] D. Jung et al., "An estimation-based task load balancing scheduling in spot clouds," in Network and Parallel Computing, pp. 571-574. Springer, 2014.

[21] V. Gulisano et al., "Streamcloud: An elastic and scalable data streaming system," IEEE Trans. Par. and Distr. Syst, vol. 23, no. 12, pp. 23512365, 2012.

[22] M.A. Rodriguez and R. Buyya, "Deadline based resource provisioningand scheduling algorithm for scientific workflows on clouds," IEEE Trans. on Cloud Comp., vol. 2, no. 2, pp. 222-235, April 2014.

[23] M. Mao and M. Humphrey, "Auto-scaling to minimize cost and meet application deadlines in cloud workflows," in Proc. 2011 Int. Conf. High Perf. Comp., Netw., Stor. and Anal., 2011, pp. 49:1-49:12.

[24] R. Shorten, F. Wirth, and D. Leith, "A positive systems model of tcplike congestion control: asymptotic results," IEEE/ACM Trans. Netw, vol. 14, no. 3, pp. 616-629, 2006

[25] P. Viola and M. J Jones, "Robust real-time face detection," Int. J. of Computer Vision, vol. 57, no. 2, pp. 137-154, 2004

[26] ImageMagick Studio LLC, "Imagemagick," http://www.imagemagick.org/.

[27] D. Lowe, "Distinctive image features from scale-invariant keypoints," Int. J. Computer Vision, vol. 60, no. 2, pp. 91-110, 2004.

[28] J. Dean and S. Ghemawat, "Mapreduce: Simplified data processing on large clusters," Commun. ACM, vol. 51, no. 1, pp. 107-113, Jan. 2008.

[29] A. Abbas, N. Deligiannis, and Y. Andreopoulos, "Vectors of locally aggregated centers for compact video representation," in Proc. IEEE Int. Conf. Multimedia and Expo (ICME'15). IEEE, 2015, pp. 1-6.

[30] A. Chadha and Y. Andreopoulos, "Region-of-interest retrieval in large image datasets with voronoi vlad," in Computer Vision Systems, pp. 218-227. Springer, 2015.

[31] J. Yang, D. Zhang, A. F. Frangi, and J.-Y. Yang, "Two-dimensional PCA: a new approach to appearance-based face representation and recognition," IEEE Trans. Patt. Anal. and Machine Intel., vol. 26, no. 1, pp. 131-137, 2004

[32] A. Garcia, H. Kalva, and B. Furht, "A study of transcoding on cloud environments for video content delivery," in Proc. 2010 ACM Multim. Workshop on Mob. Cloud Media Comput. ACM, 2010, pp. 13-18.

[33] J. Pouwelse, P. Garbacki, D. Epema, and H. Sips, "The bittorrent p2p file-sharing system: Measurements and analysis," in Peer-to-Peer Systems IV, vol. 3640 of Lecture Notes in Computer Science, pp. 205216. Springer Berlin Heidelberg, 2005.

[34] B. D. O. Anderson and J. B. Moore, Optimal filtering, Courier Corporation, 2012.

[35] R. Margolies, A. Sridharan, et al., "Exploiting mobility in proportional fair cellular scheduling: Measurements and algorithms," in Proc. IEEE INFOCOM, 2014. IEEE, 2014, pp. 1339-1347.
[36] S. Leutenegger, M. Chli, and R. Siegwart, "Optimal allocation of virtual machines for cloud-based multimedia applications," in Proc. IEEE Int. Conf. Comp. Vis. (ICCV). IEEE, 2011, pp. 2548-2555.

[37] N. Roy, A. Dubey, and A. Gokhale, "Efficient autoscaling in the cloud using predictive models for workload forecasting," in Proc. IEEE Int. Conf. on Cloud Com. (CLOUD). IEEE, 2011, pp. 500-507.

[38] V. Debusschere, S. Bacha, et al., "Hourly server workload forecasting up to 168 hours ahead using seasonal ARIMA model," in Proc. IEEE Int. Conf. on Industr. Technol., 2012.

[39] R. Calheiros, E. Masoumi, R. Ranjan, and R. Buyya, "Workload prediction using arima model and its impact on cloud applications' qos," IEEE Trans. on Cloud Comp., to appear.

[40] M. Tighe and M. Bauer, "Integrating cloud application autoscaling with dynamic vm allocation," in IEEE Netw. Oper. and Manag. Symp. (NOMS), May 2014, pp. 1-9.

[41] A. Krioukov, P. Mohan, S. Alspaugh, L. Keys, D. Culler, and R. Katz, "Napsac: Design and implementation of a power-proportional web cluster," ACM SIGCOMM Comp. Comm. Rev., vol. 41, no. 1, pp. 102$108,2011$.

[42] K. Chatfield, K. Simonyan, A. Vedaldi, and A. Zisserman, "Return of the devil in the details: Delving deep into convolutional nets," arXiv preprint arXiv: 1405.3531, 2014.

[43] A. Krizhevsky, I. Sutskever, and G. E. Hinton, "Imagenet classification with deep convolutional neural networks," in Adv. in Neural Inf. Process. Syst. (NIPS'12), 2012, pp. 1097-1105.

[44] M Hart, "Project gutenberg," 1997, http://www.gutenberg.net.

[45] Y. Kouki and T. Ledoux, "Scaling: Sla-driven cloud auto-scaling," in Proc. 28th Annual ACM Symp. on Applied Comp. (SAC'13). ACM, 2013, pp. 411-414.

[46] S Choy, B Wong, G Simon, and C Rosenberg, "A hybrid edge-cloud carcitecture for reucing on-demand gaming latency," Multim. Syst. J., vol. 20, no. 2, March 2014 .

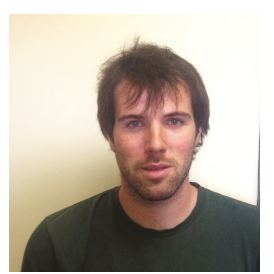

Joseph Doyle graduated from Trinity College Dublin in 2009 with a B.A.I., B.A. degree in Computer and Electronic Engineering as a gold medalist. He was awarded a Ph.D in 2013 from Trinity College Dublin. He was a post-doctoral researcher in Trinity College Dublin from 2013 to 2014. His research interests include cloud computing, cognitive autoscaling, green computing, and network optimization. He is a cofounder of Dithen Ltd. (London, U.K.) and is also Senior Lecturer at University of East London, London, U.K.

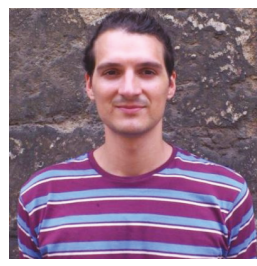

Vasileios Giotsas graduated with distinction from University College London (UCL) in 2008 with an MSc in Data Communications, Networks and Distributed Systems. He was awarded a Ph.D from University College London in 2014. He is a cofounder or Dithen Ltd. (London, U.K.) and is also a postdoctoral scientist at the UCSD Center for Applied Internet Data Analysis (CAIDA), San Diego, US. His research interests span the areas of distributed systems, cloud computing, routing protocols, Internet measurements, and Internet economics. 


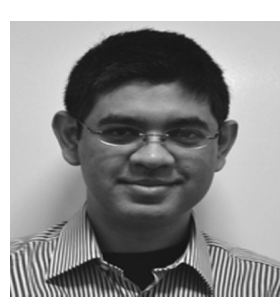

Mohammad Ashraful Anam obtained the $\mathrm{PhD}$ in Electronic Engineering from University College London (Lombardi Prize for the Best PhD thesis in Electronic Engineering) and is a cofounder of Dithen Ltd. (London, U.K.), as well as post-doctoral research associate in the Department of Electronic and Electrical Engineering, University College London, London, UK. His research interests are in error tolerant computing, and reliable cloud computing.

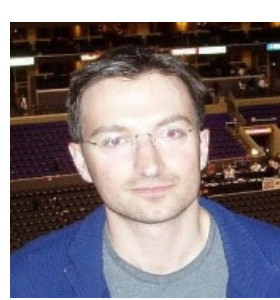

Yiannis Andreopoulos (M'00-SM'14) obtained the Electrical Engineering Diploma and an MSc in Signal and Image Processing Systems from the University of Patras, Greece, and the PhD in Applied Sciences from the Vrije Universiteit Brussel, Belgium. $\mathrm{He}$ is a cofounder of Dithen Ltd. (London, U.K.), as well as Reader (Assoc. Professor) in Data and Signal Processing Systems in the Department of Electronic and Electrical Engineering of University College London, London, U.K. His research interests are in and multimedia systems. wireless sensor networks, error-tolerant computing 